\title{
E-Mobility Systems Architecture: a model-based framework for managing complexity and interoperability
}

\author{
Benedikt Kirpes $^{1 * \dagger}$ (D), Philipp Danner ${ }^{2 \dagger}$, Robert Basmadjian $^{3}$, Hermann de Meer ${ }^{3}$ and Christian Becker ${ }^{1}$
}

\author{
${ }^{*}$ Correspondence: \\ benedikt.kirpes@uni-mannheim.de \\ ${ }^{\dagger}$ Benedikt Kirpes and Philipp Danner \\ contributed equally to this work. \\ University of Mannheim, Schloss, \\ 68131 Mannheim, Germany \\ Full list of author information is \\ available at the end of the article
}

\begin{abstract}
The future of e-mobility will consist of a large number of connected electric vehicles, smart charging stations and information systems at the intersection of electricity and mobility sector. When engineering and integrating the multitude of systems into even more complex systems-of-systems for e-mobility, interoperability and complexity handling are vital. Model-based system architectures support the engineering process of information systems with the concepts of abstraction, reduction and separation of concerns. In this paper, we contribute to the research body, by extracting requirements for managing complexity and interoperability of these systems. Further, a comparative analysis of the state-of-the-art in existing architecture models and frameworks for e-mobility is conducted. Based on the identified gaps in existing research, we propose the E-Mobility Systems Architecture (EMSA) Model, a three-dimensional systems architecture model for the e-mobility sector. Its structure originates from the well-established Smart Grid Architecture Model. We further allocate all relevant entities from the e-mobility sector to the EMSA dimensions, including a harmonized role model, functional reference architecture, component and systems allocation, as well as a mapping of data standards and communication protocols. The model then is validated qualitatively and quantitatively against the requirements with a case study approach. Our evaluation shows that the EMSA Model fulfills all requirements regarding the management of complexity and ensuring interoperability. From the case study, we further identify gaps in current data model standardization for e-mobility.
\end{abstract}

Keywords: E-Mobility, Electric Vehicles, Systems Architecture, Information Systems, Interoperability, Complex Systems, Model-Based Systems Engineering, Smart Grid Architecture Model (SGAM)

\section{Introduction}

With the advent of the "Energy Transition", the limitations of the power grid in its traditional form are becoming increasingly apparent. New requirements arise due to high amounts of power feed-in from renewable energy sources and changed consumption patterns, e.g. from self-consumption optimization and from Electric Vehicle (EV) charging processes. The resulting volatility requires fundamental changes of the electricity infrastructure to ensure future stability of the power grid. Some of the most important changes include a finer monitoring granularity in the grid and increased automation of energy management using Information and Communications Technology (ICT). In this respect,

(c) The Author(s). 2019 Open Access This article is distributed under the terms of the Creative Commons Attribution 4.0 International License (http://creativecommons.org/licenses/by/4.0/), which permits unrestricted use, distribution, and reproduction in any medium, provided you give appropriate credit to the original author(s) and the source, provide a link to the Creative Commons license, and indicate if changes were made. 
ICT has been playing a prominent role in enabling an intelligent energy management which helps in provisioning a more efficient system for generation, transmission, distribution, and consumption. Energy Informatics is playing an eminent part in cross-linking energy systems with the utilization of ICT. The Smart Grid Architecture Model (SGAM) was proposed and has soon become the de-facto reference model for the engineering and analysis of intelligent energy management systems with a special focus on power grid systems.

On the quest for realizing this energy transition and hence operating the energy system more efficiently, e-mobility is envisioned to play a fundamental role (Kossahl et al. 2012). The three former separated sectors of Electricity, Individual Mobility and ICT are merging together in future e-mobility systems. In this context, e-mobility denotes the application of electric propulsion in the daily transport sector (e.g. for people and goods). This, however, leads to novel challenges, e.g. EVs entailing an unknown demand on lowvoltage grids with potential high volatility. But it also enables synergies, for example by utilizing EVs as energy storage units or for demand-side management approaches. Thus, digitization and synergies among all sectors, not only including the energy domain (electricity, heat and mobility) but also traffic and public health, are especially important when it comes to super-ordinate coordination, like in smart cites (Neureiter et al. 2014).

Smart charging comprises concepts, enabled by ICT, which reduce the impact of EV charging processes on the grid from an energetic and economic perspective (Schmidt and Busse 2013). Besides grid-related topics, innovative business aspects such as machine-tomachine payments and transaction processing on a blockchain-based Information System (IS), require computational capability and connectivity deployed in the vehicles and in the charging infrastructure (Kirpes and Becker 2018). In order to enable such concepts, interoperable systems are required, consisting of hardware, software, processes, data, procedures and humans (ISO Central Secretary 2015).

In the fast developing field of e-mobility, harmonization of the interaction between the various systems and stakeholders, e.g. car manufactures, grid operators, energy suppliers, Charging Station (CS) operators, e-mobility service providers and EV users, is essential. In addition, new business models have to be supported by providing a map of allocated highly relevant standards, e.g. ISO 15118 and IEC 61851. Model-based systems engineering and model-driven architectures may support in handling and reducing the increasing complexity of such interoperable systems in e-mobility. A similar approach is already utilized in the electricity sector with the well-established SGAM (Dänekas et al. 2014; Neureiter et al. 2014; Neureiter et al. 2016). It supports energy domain and system engineering experts in their joint tasks to efficiently design and validate complex and interoperable smart grid systems. The SGAM constitutes a role model for the application of systems architecture models, also for other sectors. In this paper, we focus on system modelling, analysis and engineering for e-mobility and therefore consider following research question: "How does a systems architecture model need to be designed in order to ensure interoperability and manage complexity of e-mobility systems and their engineering"

The research methodology for answering this research question is based on the iterative six-step Design Science Research (DSR) process as proposed by Peffers et al. (2008), which is visualised in Fig. 1. Further, we adhere to the general DSR guidelines defined in Hevner et al. (2004). The remainder of this work is structured according to the DSR 


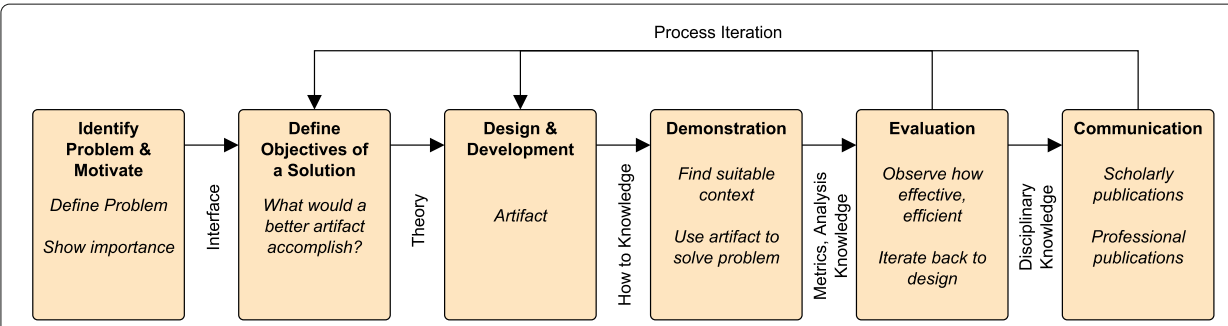

Fig. 1 Design Science Research process used for developing the EMSA framework (Peffers et al. 2008)

process as follows: First, we identify and motivate the problem in "Complexity and interoperability of e-mobility systems" section. Based on the findings from literature, these two major requirements are split into six relevant objectives of a solution. We then discuss the SGAM as a role model for tackling these challenges in the energy sector and motivate on the need for a systems architecture model for e-mobility. In "State-of-the-Art: analysis of existing e-mobility systems architecture models" section, we conduct a comprehensive analysis of related work on existing system architecture approaches. The results indicate, that all of the existing approaches have a slightly different focus, tackle different issues, and do not fully satisfy the relevant requirements of complexity and interoperability. Based on the findings and identified gaps in research, we contribute to the research body by (1) deriving design principles for a more comprehensive artifact and (2) defining the three-dimensional EMSA in "The e-mobility systems architechture (EMSA) model" section. All relevant entities are mapped to the respective layers of the model, providing a reference and guideline for adoption and implementation of the framework. Subsequently, in "Demonstration of the EMSA in the ELECTRIFIC case study" section, we show, how the EMSA can be used to solve the identified problem in a suitable context. Based on the case study in the ELECTRIFIC research project (Electrific Consortium 2019), a pragmatic observational evaluation of the artifact regarding fulfillment of the identified requirements is conducted. In the last section, the EMSA concept is concluded and we provide an outlook into future work. As typical for DSR, in this paper we provide a novel synthetic knowledge contribution to the body of research as an adaption of existing knowledge (systems architecture model) to a new problem (complexity and interoperability of e-mobility systems). Based on informal feedback and evaluation from external experts (domain and system engineering experts, who applied the framework in the context of the research project), multiple iteration cycles were conducted to design and develop our artifact. The final findings and results, specifically the proposed EMSA framework are communicated within this paper.

\section{Complexity and interoperability of e-mobility systems}

In this section, we first clarify and provide the definitions and terminology of complexity and interoperability considered in this paper. For each of the two concepts, we then extract requirements from literature. Finally, we provide the example of SGAM as a role model of the energy sector.

\section{Complexity requirements of e-mobility systems}

Complexity of systems and their engineering is a well-known challenge in the energy IS research domain (Benbya and Mckelvey 2006). The topic of complexity itself is quite 
complex and no common view or consistent definition of system complexity among various research disciplines exist. Some of the major challenges are the growing technical heterogeneity, changing requirements, human factors and multidisciplinary aspects. Two essential aspects need to be considered: 1) technical or technological complexity, and 2) organizational complexity (Lemberger and Morel 2012; Courtney et al. 2008).

The complexity of systems engineering originates in the need for expertise from different disciplines, mainly the application domain (here e-mobility) and the field of systems engineering. Experts from both fields would be the main users and beneficiaries of a better methodology. The first critical issue in the engineering process of systems for emobility is the definition of requirements, which typically reflect complex organizational problems and their transfer to a solution system (Benbya and Mckelvey 2006). Further challenges arise from the complexity of the different steps within the systems engineering process: design and modelling of the system architecture and its development.

In this paper, we refer to the terminology concerning systems architecture and related concepts as defined by ISO 42010: "The architecture of a system constitutes what is essential about that system considered in relation to its environment" (ISO et al. 2011). The system architecture is a critical element when engineering and analyzing ISs for emobility, including their sub-systems, also referred to as system-of-systems. As a first step in modelling a system, the system under study needs to be abstracted at a high level which leads to the generation of a conceptual architecture model. We extract the following main requirement and sub-requirements for a potential solution regarding complexity handling and reduction.

\section{Requirement 1: Provide means to manage and reduce e-mobility systems complexity}

- Req 1.1 : Abstraction and conceptualization Abstraction and conceptualization reduce complexity. They are typically enabled by model-based concepts and characterized by the level of abstraction. Equally important are granularity, hierarchical organization and interrelation of the concepts, systems and sub-systems (Lemberger and Morel 2012; Ranganathan and Campbell 2007). The different models can be further simplified by defining additional assumptions and limitations (Holub 2016).

- Req1.2: Separation of concerns An architecture view expresses the architecture of the system-of-interest in accordance with an architecture viewpoint" (ISO et al. 2011). Architecture viewpoints of a system are an essential concept to handle complexity by separating the concerns between and within the viewpoints. This can be achieved by considering different dimensions, layers or tiers, as well as de-coupling and simplifying each of them (Holub 2016; Lemberger and Morel 2012).

- Req 1.3 : Re-usability of components Components on different layers (sub-systems, concepts, roles, services) should be re-usable in order to reduce complexity (Lemberger and Morel 2012). Re-usability can be ensured by harmonization (e.g. due to role models or technical homogeneity) and standardization (e.g. by utilizing standardized notations such as Unified Modeling Language (UML) and Systems Modeling Language (SysML)). 
- Req 1.4: Complexity reduction of the engineering process

The complexity of the overall engineering process can be reduced by simplifying each of its phases: requirements, design, development, verification and validation (King and Simon 2015; Honour 2006; Benbya and Mckelvey 2006). A visual representation of the views on the model, supports the engineering process (Uslar et al. 2019).

Guidance through the whole process and documentation of the tool provide support with application and execution.

\section{Interoperability requirements of e-mobility systems}

In order to successfully integrate EVs with existing and upcoming smart grid, mobility and energy systems, interoperability and standards play a vital role (Brown et al. 2010). The IEEE defines interoperability as "the ability of two or more systems or components to exchange information and to use the information that has been exchanged" (Geraci et al. 1991). Consequently, interoperable systems are able to provide and support services between each other. However, this typically requires common standards. Connectivity and compatibility are weaker states than interoperability. If one system is dominant and does not depend on open standards, a second system adapted to work with this first system, is only compatible and not interoperable. According to the GridWise Architecture Council (GWAC) interoperability stack (GWAC 2008), interoperability can be defined on three levels: organizational (pragmatics), informational (semantics), and technical (syntax). We extract following main requirement and sub-requirements for a potential solution regarding interoperability.

\section{Requirement 2: Provide means to analyze and ensure e-mobility systems interoperability}

- Req.1: Analysis and assessment of interoperability Interoperability should be analyzed and ensured on all three levels (organizational, informational and technical) of a system within its environment and between its sub-systems (GWAC 2008). Due to the standardization aspect of this requirement (Brown et al. 2010), it is closely connected to Req1.3 (Re-usability of components). The analysis of interoperability is important for new, but also for existing systems and sub-systems.

- Req2.2: Identification of standardization gaps A comprehensive solution approach for handling interoperability of system architectures should also enable the identification of gaps in standardization. Based on the interoperability analysis as defined in $\operatorname{Req}_{2.1}$ it should become clear, whether an identified interoperability issue is either a shortcoming of the current system, or a gap in the overall standardization.

Various solution approaches exist to potentially tackle these requirements for emobility systems, e.g. Architecture Description Languages, Model-based systems engineering (Lopes et al. 2011) in combination with a Domain-Specific Language (DSL) (Neureiter et al. 2016) or the Model-Driven Architecture (MDA) defined by the Object Management Group (Object Management Group 2014). In this paper, we focus on systems architecture models which constitute a suitable solution for the identified problems and are highly desirable as a common frame for reference (Uslar and Engel 2015). 
The Smart Grid Architecture Model (SGAM)

Regarding the handling of complexity and ensuring interoperability of cyber-physical systems with systems architecture models, the electricity sector acts as a role model for e-mobility. Following a standardization request of the European Commission in 2012, the SGAM has been proposed as a reference architecture model for smart grid systems (CENCENELEC-ETSI Smart Grid Coordination Group 2012). This framework can be used to design, engineer, visualize and validate smart grid architectures and to analyze smart grid use cases and systems regarding interoperability and standardization gaps in a structured way. The SGAM has a strong European focus and has been applied in several European R\&D projects (European Commission 2019) such as FP7 DISCERN, FP7 ELECTRA, FP7 EcoGrid EU, H2020 SmartNet and H2020 Flexiciency. Further, many research institutions such as RWTH Aachen, OFFIS Oldenburg, TU Delft or KTH Stockholm, consider the SGAM as part of their energy systems research and education. An exhaustive overview about the usage of SGAM in research is given in Uslar et al. (2019). In industry, it is adopted and used for practical applications regarding visualization, validation and configuration of smart grids by multiple organizations, e.g. Siemens Infrastructure and Cities, Accenture Consulting, City of Mannheim or the German Energy Agency (dena).

The SGAM consists of three dimensions (see Fig. 2): interoperability layers, zones and domains. The five smart grid interoperability layers are extracted from the GWAC interoperability stack (GWAC 2008).

The business layer represents the business viewpoint on the information exchange in a smart grid, e.g. regulatory and economic (market) structures, business models, busi-

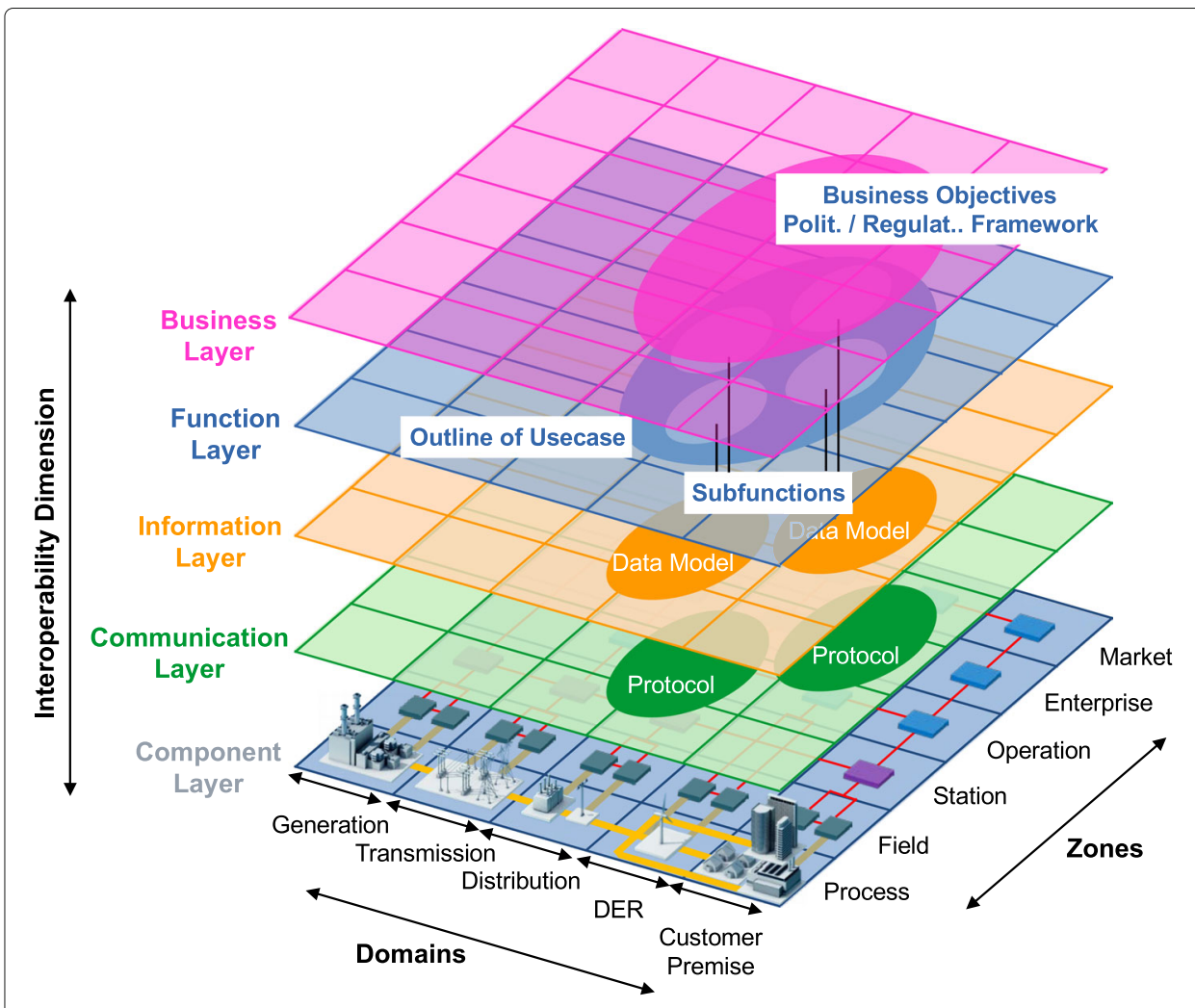

Fig. 2 Smart Grid Architecture Model (from CEN-CENELEC-ETSI Smart Grid Coordination Group (2012)) 
ness processes and business cases of stakeholders. The function layer describes system use cases, functions and services including their relationships from an architectural viewpoint, independent from actors and physical implementation of applications, systems and components. Both, business and function layer implement the organizational (pragmatics) level from the GWAC stack, including economic/regulatory policy, business objectives and business procedures. The informational (semantics) level, including business context and semantic understanding, is mapped to the information layer of the SGAM. It describes the information flow and the corresponding information objects that are exchanged between functions, services and components and their underlying canonical data models, representing the common semantics for an interoperable information exchange. The communication layer describes communication protocols and technologies for the interoperable exchange of information between components in the context of the underlying function and related information objects or data models. The component layer represents the physical components in the smart grid. These include systems and devices, power system equipment (typically located in the process and field zone), network infrastructure and any kind of computational hardware. The communication and the component layers implement the GWAC technical (syntax) level, including aspects such as syntactic interoperability, network interoperability and basic connectivity.

The zones (process, field, station, operation, enterprise, and market) reflect the hierarchy within power systems management and the domains (generation, transmission, distribution, distributed energy resources, and customer premises) represent all steps in the energy conversion chain. Further details about the considered zones and fields are given in "The E-Mobility Systems Architecture (EMSA) model" section of this paper.

Within the energy sector, the SGAM is highly relevant, both for research and industry. It supports the main users, systems engineering and domain experts in better fulfilling their tasks such as visualizing, modelling, designing, engineering and analyzing complex and interoperable smart grid systems. systems architecture models like the SGAM are comprehensive and efficient concepts to tackle complexity and interoperability requirements of such systems. The e-mobility sector faces similar challenges of increasing complexity on all layers and the need for interoperability. Therefore, we limit potential solution approaches to systems architecture models for e-mobility by considering the identified requirements.

\section{State-of-the-Art: analysis of existing e-mobility systems architecture models}

In this section, we provide a comparison of related work on systems architecture models and frameworks in the e-mobility sector (Table 1). We analyze and discuss the state-of-the-art regarding the scope of the respective approach, its structure, allocation of entities to the dimensions, applicability for pure e-mobility or sector-coupled systems and whether their usefulness has been demonstrated and evaluated. Further, the fulfillment of both requirements, complexity reduction and interoperability analysis, are shortly assessed. Based on this analysis, we identify gaps in the existing research body and in the next section, derive design principles for our artifact.

A promising approach to tackle the complexity of sector-coupled systems (e.g. for smart cities), is the Generic Smart City Architecture Model (GSCAM) proposed by Neureiter et al. (2014). This framework focuses on the multiple sectors of a smart city. It can be 


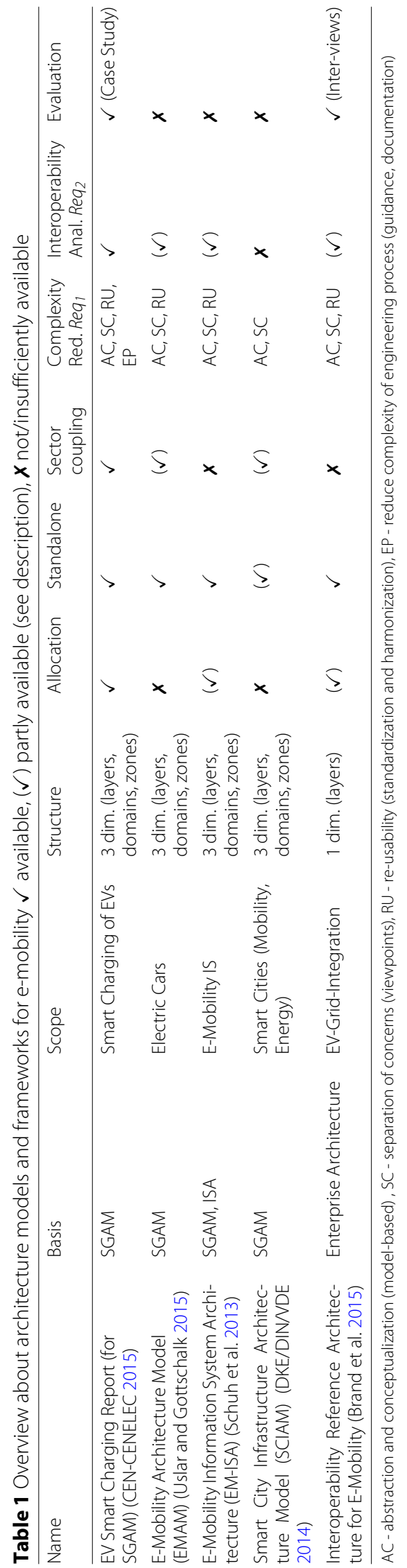


used to consider multiple application domain cubes parallel to the SGAM, in order to handle complexity and interoperability on a larger scale and broader context. We consider GSCAM as an extension of SGAM, which could be used to model and depict sector coupling of systems (column Sector coupling in Table 1).

\section{E-mobility in the SGAM}

The SGAM has initially not been designed for an extensive application with e-mobility use cases and systems. Consequently, the e-mobility process chain does not perfectly fit to the SGAM's grid-specific domains, which are designed to only represent the energy conversion chain. E-mobility additionally requires an interconnection of multiple domains such as charging infrastructure (e.g. electrical and hydrogen CSs), (mobile) EVs and especially human machine interfaces for the users with mobility-focused use cases.

In a technical report of CEN-CENELEC about EV smart charging (CEN-CENELEC 2015), first steps have been taken to map parts of the e-mobility sector onto the SGAM. This approach considers specific battery charging-related systems (e.g. smart charging with relevant systems, standards and protocols) as scope, while e-mobility in general is not tackled adequately. Considering the EV as enabler of e-mobility (= power grid as enabler of electricity in SGAM) and the EV user as consumer of e-mobility (= consumption devices in SGAM), the EV user interactions are only modelled through its respective EV in SGAM. As another example, CSs have been allocated multiple times in SGAM, once in the Distributed Electrical Resource (DER) and once in the Customer Premise domain, which might lead to confusion. Due to the view on mainly energy-specific domains, the structure is also not ideal for e-mobility. The majority of use cases and systems of the emobility ecosystem are located in the distribution, DER and customer premises domains. From the e-mobility point-of-view, this leads to a reduction of details in the separation of systems and actors to the corresponding domains.

The SGAM is applicable for standalone usage but could also be used for sector-coupled systems (e.g. within the GSCAM (Neureiter et al. 2014)). The relevant requirements for interoperability and complexity are sufficiently fulfilled. The smart charging use cases have been evaluated with a case study focusing on grid-related topics (e.g. voltage control), demand-side flexibility management and different charging actions (e.g. uncontrolled or demand-response charging).

The SGAM is well established for the usage in smart grid domains and some architecture models for e-mobility exist, which adhere to the fundamental SGAM structure by utilizing a three-dimensional approach with layers, domains and zones. Typically, these models have a very strong focus on grid integration and consider e-mobility as a sub-discipline of electricity. According to Uslar and Gottschalk (2015), the challenge for SGAM-related frameworks is the definition of a useful and appropriate sector-specific structure mainly on the zones and domains dimension.

\section{EMAM}

The EMAM is an SGAM-based approach, which has been introduced as work-inprogress by Uslar and Trefke (2014); Uslar and Gottschalk (2015). The three-dimensional EMAM has its scope mainly focused on electric cars and is intended to be used standalone. The interoperability layer dimension is the same as in SGAM, while zones (in-car, plug, cable, pole, pcc, grid) and domains (distribution, DER, building, home Energy 
Management System (EMS)/human machine interface, EV battery) have been adjusted to better fit the conductive charging process of battery electric cars.

Definition and description of domains and zones are missing or are very elementary. Specifically, the zones dimension is lacking the hierarchical organizational granularity and data aggregation aspects which are used in the SGAM zones. The proposed zones more describe the actual process chain of charging an EV, thus would better fit on the domain axis. In the EMAM, Sector coupling can only be modelled for the electricity sector, since the zones dimension is incompatible with the GSCAM approach. Interoperability is addressed on business (harmonization), information and communication layers (standardization), but no allocation of standards to the model is included. Standards and roles are described but not mapped and allocated to the model dimensions. This makes the EMAM unsuitable for interoperability assessment. Complexity handling is similar to the SGAM, but much more concise with guidance and documentation completely missing. Further, no evaluation has been conducted.

\section{EM-ISA}

Schuh et al. (2013) also identify the need for interoperability and propose the domainspecific EM-ISA. It is extracted from a collection of generic IS architectures and mapped onto an adaptation of the SGAM. It provides guidelines for the architecture design of IS supporting different business models in e-mobility. The EM-ISA changes the naming of the information layer to data layer and component layer to element layer, considering the user as actor and hence violating the separation of concerns.

For the zones, process and field have been removed, the domains are distinguished in immobile/infrastructure (CS, parking, IT back-end), mobile/user of infrastructure (EV, user, user device). This makes it incompatible to the GSCAM and only usable standalone. For harmonization purposes, meta models (e.g. for functions) are included, but an allocation of the entities to the EM-ISA structure has been done only partially. Interoperability is not considered in more detail, no standards are mentioned. Also no evaluation has been conducted.

\section{SCIAM}

Another approach is the SCIAM which has been shortly introduced as a draft by the German standardization road map smart city (DKE/DIN/VDE 2014). Its focus is not purely on e-mobility but the model considers e-mobility as part of electricity and mobility sector in a smart city. The architecture model is intended to be used standalone, but e-mobility is only considered at the intersection between mobility and electricity. The SCIAM itself considers sector coupling, but is not compatible with the GSCAM framework. No further information on this architecture model has been provided.

\section{Interoperability reference architecture for e-mobility}

The interoperability reference architecture for e-mobility (Brand et al. 2015) is intended to support the integration of EVs into the electricity system. Its one-dimensional structure consists of four layers (business, business services, application, and infrastructure). However, it supports complexity and interoperability handling only on conceptual level without providing any specific standards. As it is not based on SGAM, compatibility with GSCAM is not given. Relevant entities have been allocated to the respective layers. It can 
be used standalone and acts as a reference architecture for interoperable e-mobility, but is not directly intended for the engineering of complex systems as it provides no tools or guidelines. The validity of the reference architecture has been evaluated with expert interviews.

\section{Analysis results}

As shown (with an overview in Table 1), most of the state-of-the-art approaches lack the scope of a comprehensive e-mobility ecosystem, that not solely focuses on charging via a conductive connection. Only the technical report on smart charging of EVs in the SGAM includes an adequate allocation of existing standards. All models can be used standalone, but sector-coupling is not their focus and only possible with some of the concepts to some extent. Most of the approaches are not comprehensive, missing guidance for application and an evaluation. The main requirements regarding complexity reduction and interoperability assessment are not satisfactorily met and thus, a new systems architecture model based on the advantages of the state-of-the-art needs to be introduced.

\section{The E-Mobility Systems Architecture (EMSA) model}

In this section, we describe the final artifact design (Basic Design Principles for the EMSA Model) and its definition (Development of the EMSA Model). Multiple iterations and feedback cycles were conducted, considering informal feedback from discussions with system engineering and domain experts, who applied the model and framework during the three-year ELECTRIFIC project for multiple systems.

\section{Basic design principles for the EMSA model}

From the analyzed systems architecture models, we extract principles for the design of our artifact. These constitute the guidelines for the development of the e-mobility systems architecture model. The first three design principles are directly extracted from the related work analysis. Design principles four and five are more generic and stem from the basic principles for the domain-specific SGAM.

Design principle 1: Scope and applicability The systems architecture model is intended to be comprehensive and cover the complete scope of the e-mobility sector. It should be applicable for standalone usage, considering use cases and systems, which are not related to any other sectors. At the same time, it should also be applicable for systems that affect multiple sectors (e.g. compatible with the GSCAM approach).

Design principle 2: Multi-dimensional structure The systems architecture model is intended to provide an appropriate number of useful sector-specific dimensions. For a model, which is based on the SGAM and compatible with the GSCAM, the ideal structure also consists of three dimensions: interoperability layers, domains and zones. Contiguity (either geographical, hierarchical or logical) of all dimensions along each axis is essential. Only limited aspects of the domain value chain should be changed, providing a clear domain abstraction (Uslar and Gottschalk 2015).

Design principle 3: Allocation, localization and consistency The fundamental idea of the systems architecture model is to provide an appropriate allocation of all e-mobility 
entities to its structure. Across all dimensions, the appropriate location for each entity should be identified and specified. By adhering to this principle, all entities and their relations can be represented in a clear systematic and comprehensive view. Further, a consistent mapping is essential in order to be able to identify gaps in specifications or inconsistencies in the system (CEN-CENELEC-ETSI Smart Grid Coordination Group 2012).

Design principle 4: Universality and flexibility The systems architecture model is intended to represent e-mobility architectures in a common and neutral view. It should be technology-agnostic and not give any preferences to existing architectures. Obtaining flexibility on all layers supports alternative use cases, system designs and implementations. It further supports future advancements and enables concepts like scalability and extensibility (CEN-CENELEC-ETSI Smart Grid Coordination Group 2012).

Design principle 5: Extensibility and scalability The systems architecture model can be extended with additional entities or even by adding new domains and zones, when the sector evolves (compatibility with the SGAM needs to be maintained). The systems architecture model can be scaled up to a top-level view of the whole e-mobility ecosystem or scaled down to a specific and very detailed subset of use cases, functions and systems (CEN-CENELEC-ETSI Smart Grid Coordination Group 2012).

The EMSA is developed considering the above mentioned five design principles. In order to apply some of the SGAM framework's tools, it is recommended to only alter aspects of the domain dimension (Uslar and Trefke 2014). This principle is also in line with the GSCAM approach (Neureiter et al. 2014). Subsequently, the EMSA is defined in a way, that is compatible with the GSCAM but also usable as standalone architecture model. Cross-cutting issues, that cannot be allocated explicitly to one domain or one zone like telecommunication systems and security, need to be represented separately and are not considered in this work (CEN-CENELEC-ETSI Smart Grid Coordination Group 2012).

\section{Development of the EMSA model}

In the following, the structure of the EMSA Model including all relevant domains along the e-mobility process chain will be discussed. For the EMSA scope, e-mobility is not limited to battery electric vehicles, but also includes all kind of other vehicles with an electric drive train, for example electric tram or hydrogen trucks with fuel cells. The dimensions of the EMSA Model are visualized in Fig. 3. In order to obtain a maximum level of compatibility to GSCAM and SGAM, both the number of layers (five business, function, information, communication, component) and zones (six - process, field, station, operation, enterprise, market) in the EMSA Model are kept the same as in SGAM. The definition of the zones is adapted to be more appropriate for the context of e-mobility.

\section{Definition of domains}

Similar to the energy supply chain in SGAM, the whole e-mobility process chain is represented on the domain axis and split into different domains. Inspired by Schuh et al. (2013), the proposed domains are also classified as immobile (Energy Conversion, Energy 


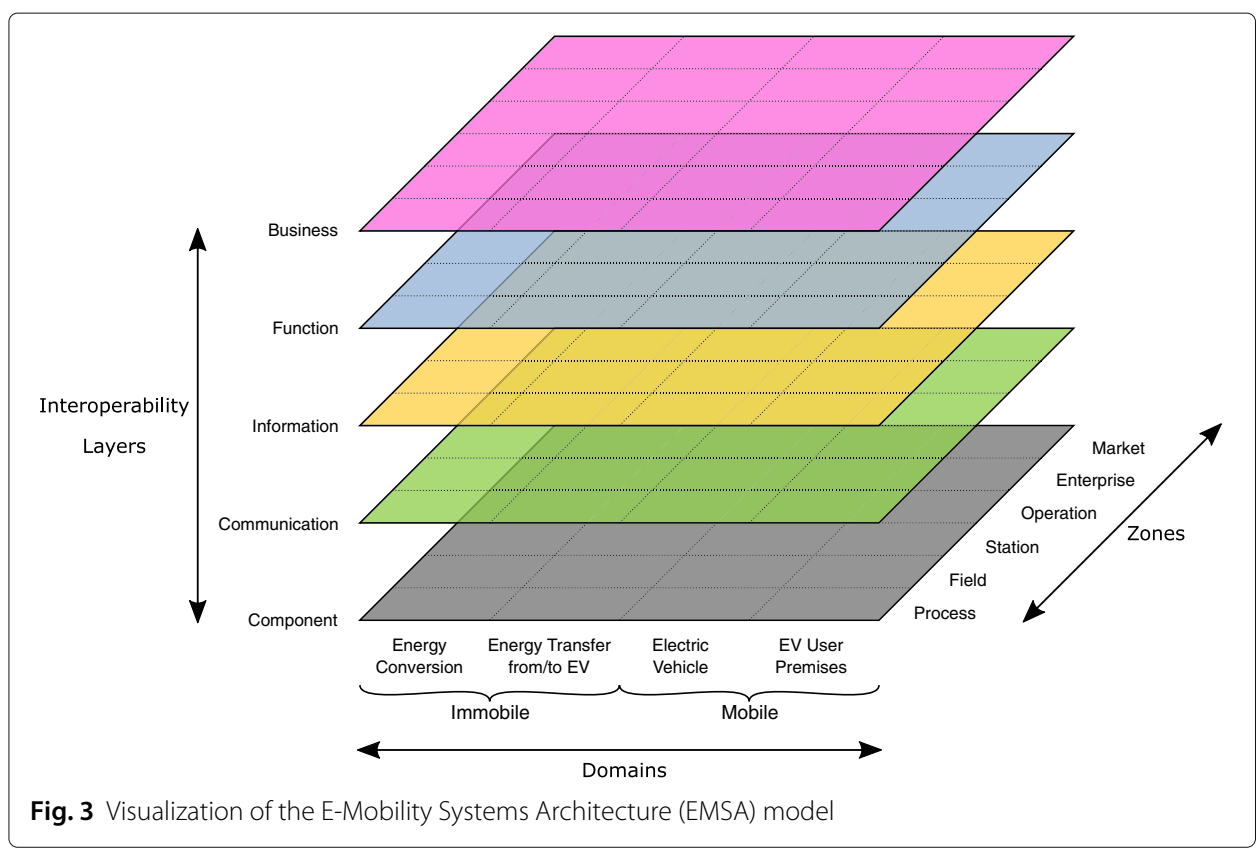

Transfer from/to EV) and mobile (Electric Vehicle, EV User Premises). Next, we provide a definition for each domain by giving specific examples:

- Energy Conversion includes energy sources and the energy conversion chain. This contains the electricity system with all levels including generation, transmission grid, distribution grid and local power generation like photo-voltaic systems. It can also represent energy from other sources that is later transformed into electrical energy, like hydrogen fuels that may be generated locally or transported via a piping system.

- Energy Transfer from/to EV includes the necessary infrastructure for transferring the energy to the EV and vice versa. As example, CSs, catenary wires for trains or hydrogen fuel stations can be listed. In addition, the CS management system and all kind of entities required for the process of getting energy to/from the EV, like vehicle-to-vehicle, vehicle-to-grid, grid-to-vehicle, vehicle-to-home or home-to-vehicle, are included.

- Electric Vehicle includes the entities to perform the electric driving process. This includes e-bikes, e-scooters, e-cars, e-buses and e-railway. In addition, all components and systems, that are part of the moving EV, like the battery, Battery Management System (BMS) or monitoring systems as well as EV (fleet) management systems are part of this domain.

- EV User Premises includes interfaces for the end users like mobile devices, personal computers or (RFID) charging cards. This could be an interface for the purpose of managing the EV (e.g. smartphone app for EV preconditioning) or searching/booking/reserving CSs or vehicles, e.g. train and car-sharing. In addition, 
intelligent route planning, navigation and all kind of e-mobility services for end users are located in this domain.

\section{Definition of zones}

The zones represent the hierarchical levels of e-mobility management and use the concept of aggregation and functional separation. Concerning the aggregation, one can distinguish between data aggregation (e.g. data concentrated from field to station zone) and spatial aggregation (from distinct location at field and station to wider area at operation, enterprise and market). Functional separation is given, inter alia, by the spatial aggregation, as local functions, like in-car communication or protection equipment in the CS or in the grid, are mainly implemented in the field and station zones. The same applies for more global functions like monitoring or billing, which are located in the zones operation, enterprise and market. We therefore define the zones of the EMSA Model similar to the well-defined zones in SGAM in order to also ensure compatibility with the GSCAM framework. However, the definition has been adjusted to better fit the e-mobility sector:

- Process includes the physical or chemical transformation of energy (electricity, hydrogen fuel, etc.), the information flow in all domains, and all directly involved physical equipment. This can be entities of the power grid, CSs, EVs, end user devices or any kind of sensors and actuators which are directly associated with the e-mobility process.

- Field includes equipment to protect, control, monitor and support the process of e-mobility such as (1) protection relays at a CS, power grid or in the EV, (2) metering devices and any kind of intelligent electronic devices which acquire, process and use related data like the RFID authentication method.

- Station represents the areal aggregation for the field zone, e.g. for data concentration, functional aggregation or local sensor systems. An aggregation level could be a charging spot with multiple CSs or the internal communication and control system of an EV (e.g. in-car Ethernet, FlexRay or CAN bus).

- Operation hosts management entities in the respective domain for the processing of aggregated data, e.g. Local or Grid Energy Management System, EV Management System, CS Management System, Human Machine Interface Devices for input from the user or data provision services.

- Enterprise includes commercial and organizational processes, services and infrastructures for enterprises (utilities, service providers, energy traders, etc.), such as asset management, logistics, work force management, staff training, customer relation management, billing and procurement.

- Market reflects the market operations possible along the e-mobility chain, e.g. charging service networks, e-mobility provider services, EV sharing, energy trading, as well as (user) data trading platforms.

\section{EMSA interoperability layers}

One methodology for complexity handling is the separation of concerns, which can be realized by considering different architecture viewpoints such as business, functional 
or communication architecture, here implemented as layers. On each layer, various standardization and harmonization means are implemented to ensure interoperability and enhance re-usability. Further, all relevant entities are appropriately allocated to the EMSA dimensions (layers, domains, zones).

EMSA business layer On the business layer, different economic and legal aspects of the business architecture can be modelled, e.g. business cases, business services, business processes, business models and regulatory constraints. Harmonization and abstraction are the major constituents to handle complexity on this layer. Besides standardized notation languages such as UML, a harmonized business actor role model is essential. The most important business actor roles in the domain of e-mobility, compiled from different standards (Open Charge Alliance 2018; Nationaal Kennisplatform Laadinfrastructuur et al. 2019; ISO Central Secretary 2016), are shown and allocated to the EMSA domains (not considering zones) in Fig. 4.

The EV user can be differentiated into private EV Owner and EV Fleet User (e.g. a taxi driver). The latter one is using an EV that is owned by the EV Fleet Operator. The Equipment Provider sells or leases equipment (e.g. EV or battery) to EV Owner and EV Fleet

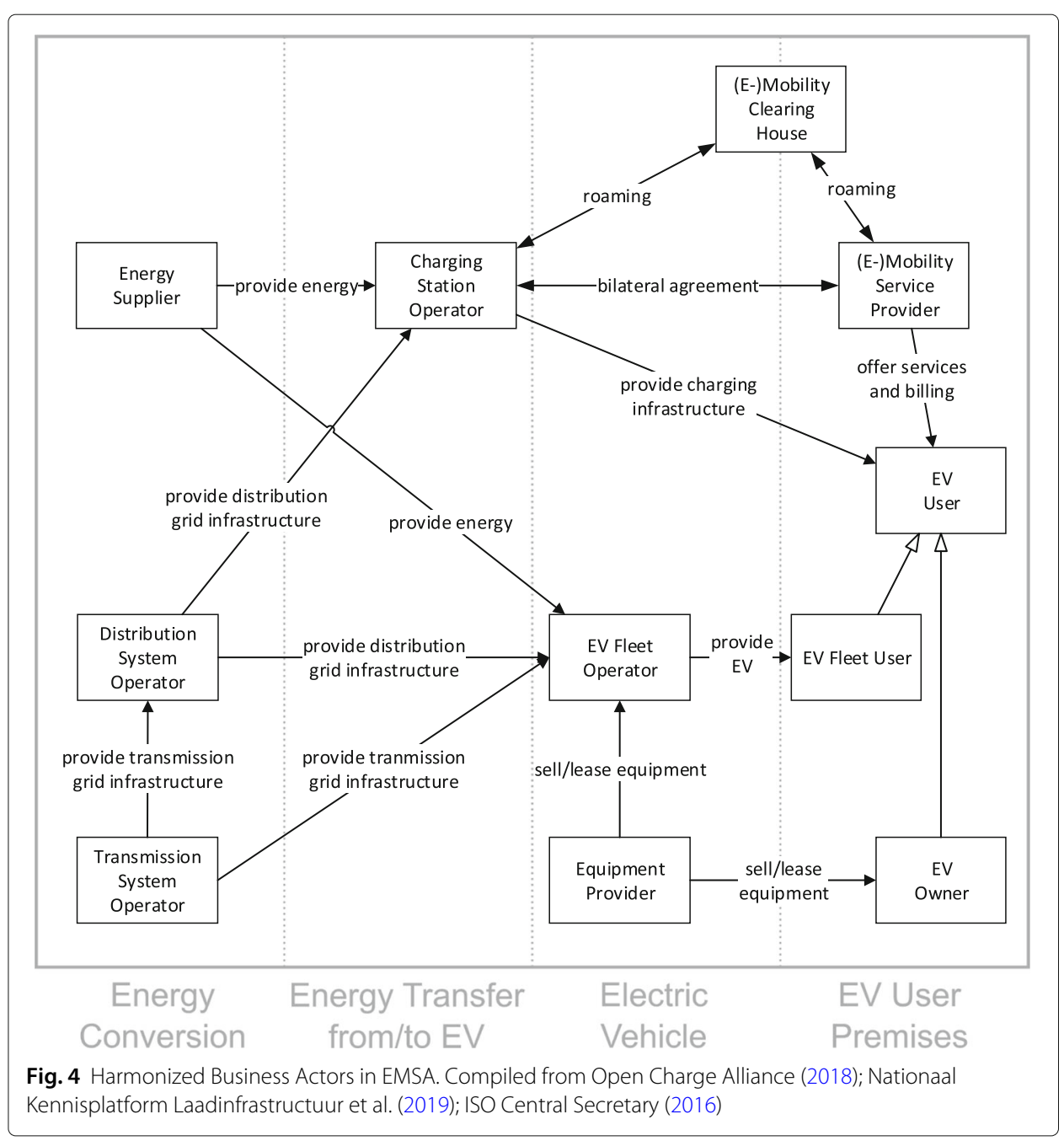


Operator (e.g. public bus transport). In the energy conversion and transfer domains, three major roles exist. The Power Grid Operator (typically the Distribution System Operator (DSO) or Transmission System Operator (TSO)) provides the grid infrastructure either directly to the EV fleet operator (e.g. catenary for trains) or to the CS operator. Both actors are provided with electricity from the Energy Supplier. The CS operator provides charging infrastructure to the EV user and enters into a bilateral agreement with the EMobility Service Provider. The E-Mobility Service Provider offers services to the EV user and also handles the billing process. Payment transaction settlement is done via roaming by a Clearing House.

EMSA function layer The function layer describes the functional architecture and elements of the system. It connects business cases with their physical implementation by an abstraction of interconnected functions. The interactions of the functions indicates required information exchange between them. Depending on the level of abstraction, the functions can be described, grouped and clustered differently. In Fig. 5, the most relevant high-level function groups of e-mobility sector (extracted from CEN-CENELEC (2015)) are allocated to the EMSA function layer. The functional architecture can be detailed, e.g. by utilizing UML activity or sequence diagrams.

EMSA component layer The component layer is the basis for the upper four layers. In Fig. 6, the component layer of the EMSA Model and the most relevant systems and

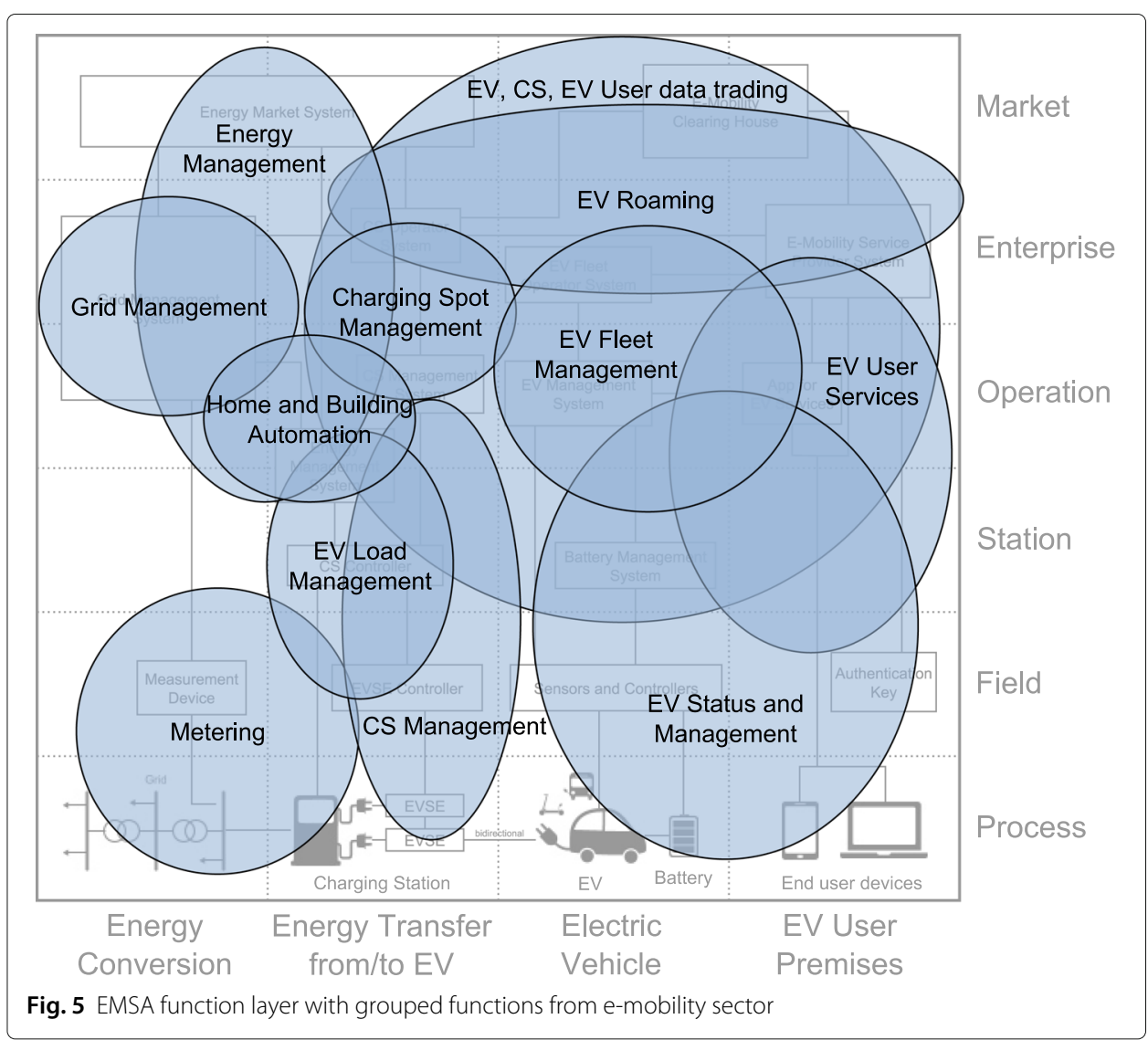




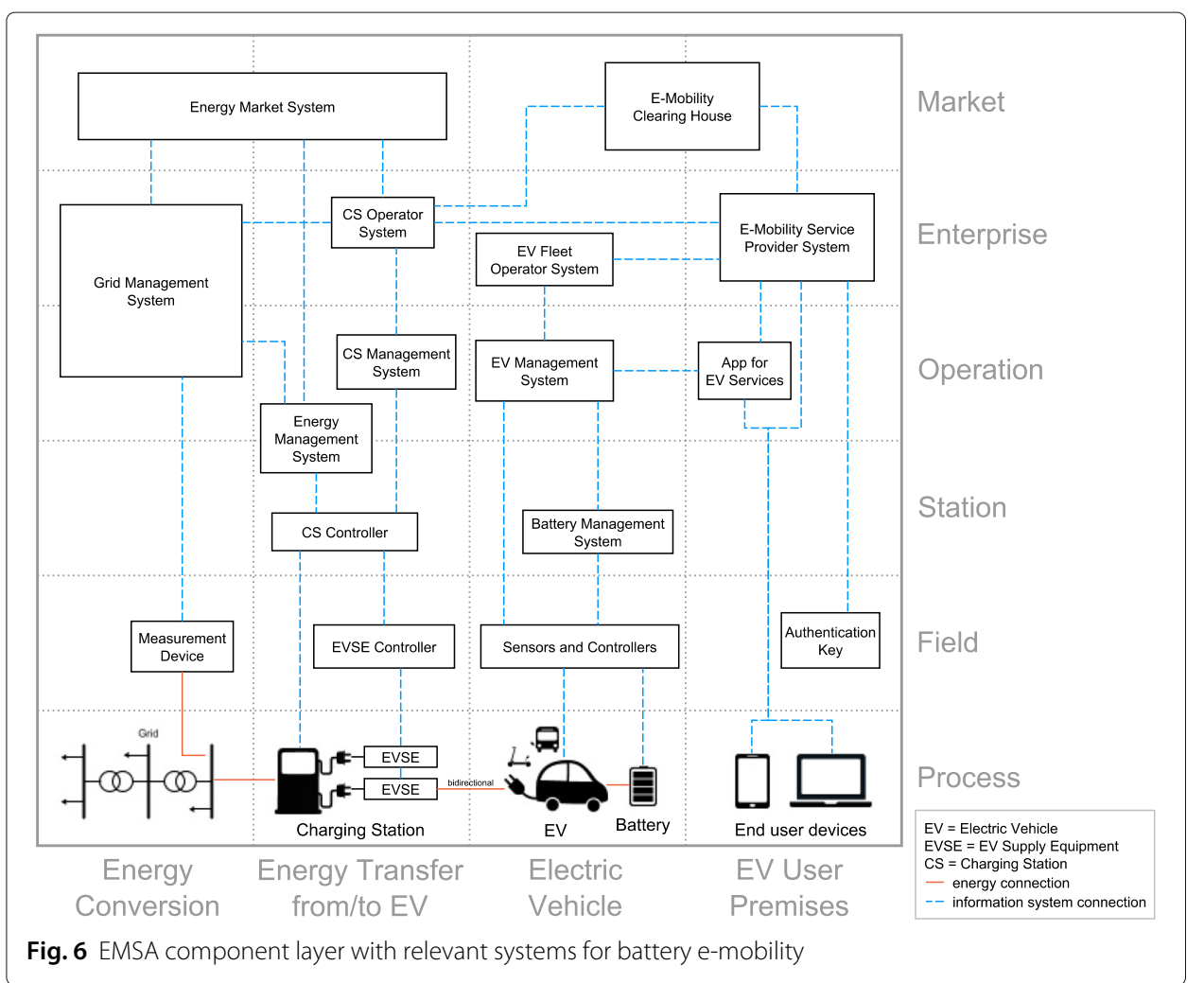

hardware/software components for battery-electric mobility are shown. The components for non-battery e-mobility are excluded for reasons of clarity and comprehensibility. To comply with the case study in the validation section, here the focus is limited to batteryelectric mobility.

EMSA information layer The information layer is closely linked to the communication layer. The focus of the information layer is on the three aspects of data management, integration concepts and the required information exchange interfaces. Standardized information flow and data models between services are important for homogeneous connected sub-systems, ultimately leading to interoperability of the whole complex systemof-systems. In Fig. 7, the most relevant standards and protocols for the e-mobility sector, in specific for battery-electric mobility, are categorized. The protocols are extracted from ElaadNL (2017); Rodríguez et al. (2015); Schmutzler et al. (2013). This allocation helps in identifying gaps in standardization.

Various standards from IEC, ISO, ETSI, ITU, IEEE and SAE can be mapped to the different zones and domains. In the area of grid management, the IEEE 2030.5 (Adoption of Smart Energy Profile), the IEC 61850 family and OpenADR (IEC 62746-10-1) can be mentioned. For the information exchange between the Grid Operator and the CS operator, OSCP (Portela et al. 2015) or the Flexibility Reward Scheme (Danner et al. 2018) deliver solution to adjust the EV charging processes to the needs of the power grid. Information exchange protocols related to energy markets are for example OASIS EMIX, IEC 62325, EN 62325-301/351 (Entso-e MADES). The information exchange between car and CS could be handled with IEC 15118 or IEC 61851-1. The information exchange 


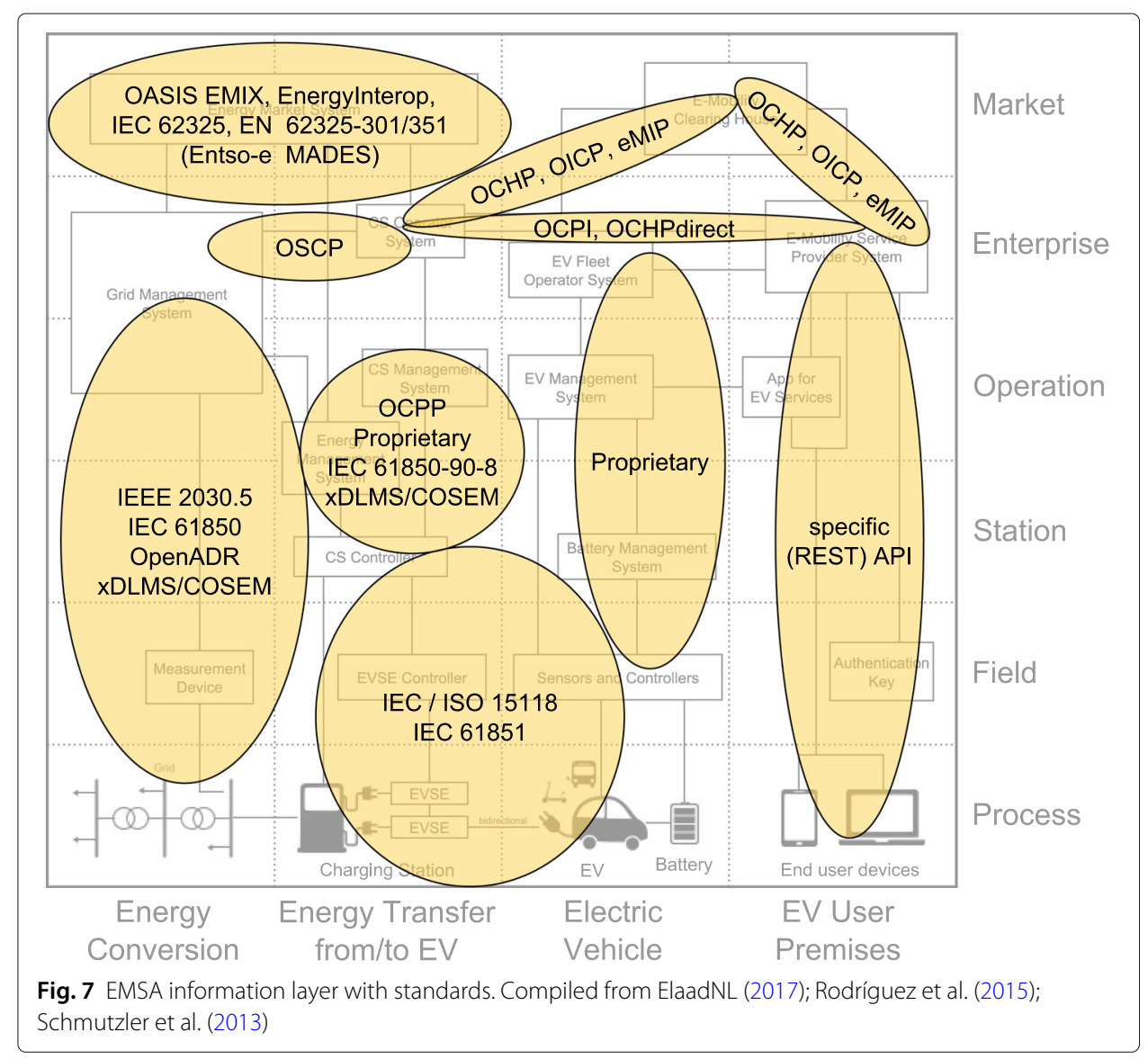

and control from the CS management system or an Energy Management System to the CS is usually done with OCPP. To handle information exchange between the CS operator and the E-Mobility Service Provider, OCPI or OCHPdirect could be used within a direct communication or OCHP, OICP or eMIP using an E-Mobility Clearing House as mediator.

EMSA communication layer The main objective of the communication layer is to visualize the communication infrastructure (protocols, technology) and identify gaps in the existing communication standardization, or to show lack of standards implementation in the respective system. Therefore, we allocated existing and commonly used protocols, extracted from CEN-CENELEC (2015); ElaadNL (2017); Rodríguez et al. (2015), to the corresponding domains and zones. The result is shown in Fig. 8 and indicates no major gap for communication standards. In high level zones (operation, enterprise and market, as well as, for the communication with end user devices), usually Web Services, HTTP over SSL and TCP/IP are used. For energy markets the IEC 61968-100 and for general market places EN82325-450/451 can be mentioned. Communication in the field of metering and grid management usually uses domain-specific protocols, e.g. the IEC 62056 xDLMS/COSEM for smart meter communication in general and CLC/prTS 505685 for smart meter data exchange communication are relevant, for their communication with higher zones usually ISO 9506 (MMS) from the IEC61850 standard family comes into play. Concerning communication from EV to CS either Pulse Width Modulation 


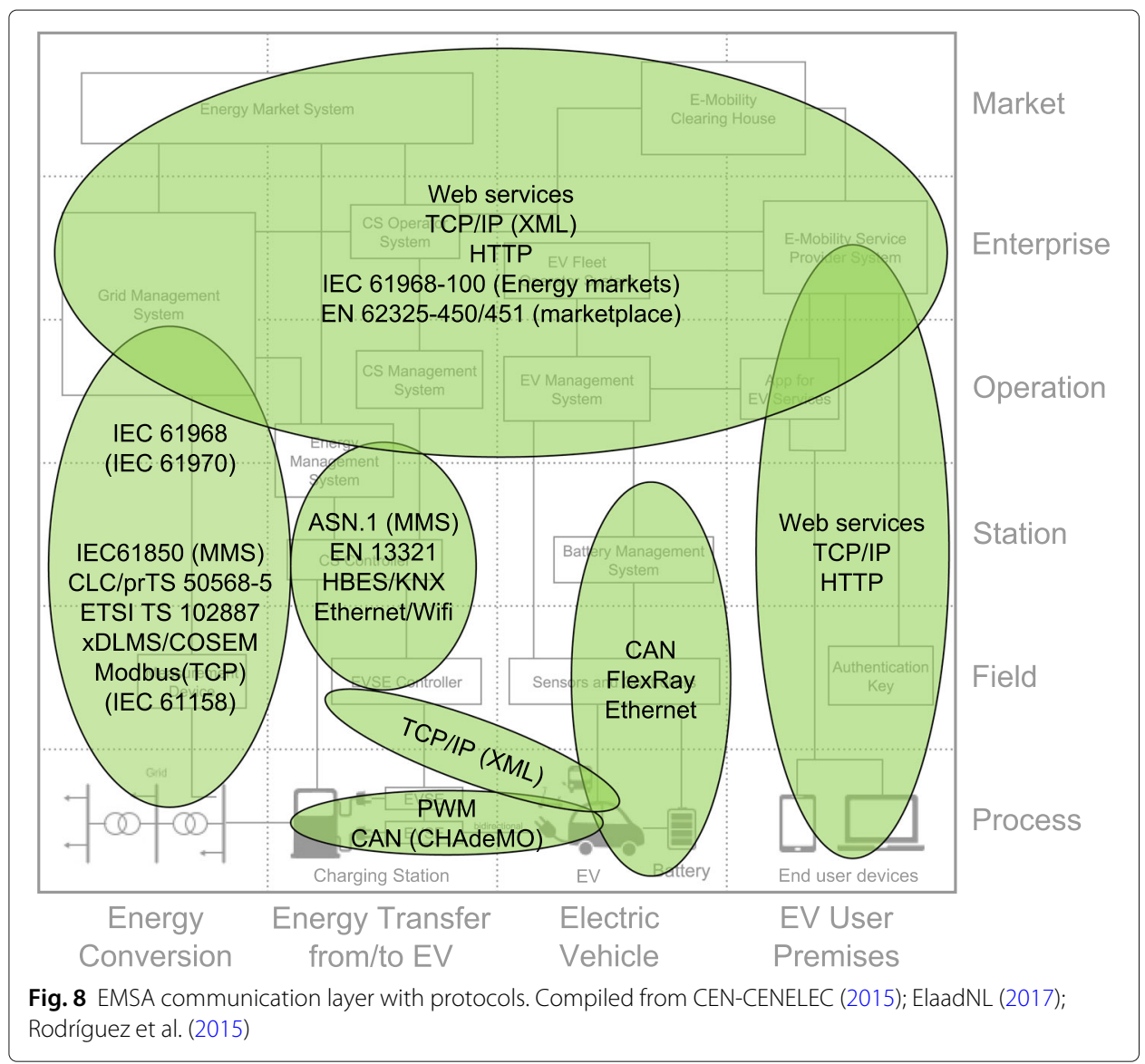

(PWM) signals according to IEC61851-1 (SAE J1772) or the newer communication standard ISO/IEC 15118-2 XML (EXI) TCP/IP can be utilized. CHAdeMO uses two CAN buses for communication to the EV. Charging spots can use ETSI TS 101556 ASN.1 and future standards for OCPP/OCHP. For in-car communication, typically the ISO 11898 CAN bus or more recently the former industry standard FlexRay (now ISO 17458-1 to 17458-5) or Ethernet (ISO/DIS 8802/3) are used for sending information between the different components.

\section{MDA approach for the EMSA model}

In the smart grid domain, MDA as a sub-discipline of Model-Driven Engineering is a suitable approach for handling systems complexity in combination with the SGAM (Uslar et al. 2019; Dänekas et al. 2014). We apply this methodology and re-use this mapping to propose a similar approach for our EMSA Model (represented in Fig. 9). The MDA concept is intended to foster separation of concerns by separating the business and functional architecture from a specific technological implementation (Object Management Group 2014). Mapped to the EMSA Model, the Computational Independent Model is defined in the System Analysis Phase on business and function layer. The Platform Independent Model is specified during the System Architecture Phase on component, information and communication layer. In the last phase, the Design and Implementation Phase, the Platform Independent Model is transformed into a Platform Specific Model and implemented as Platform Specific Implementation. 


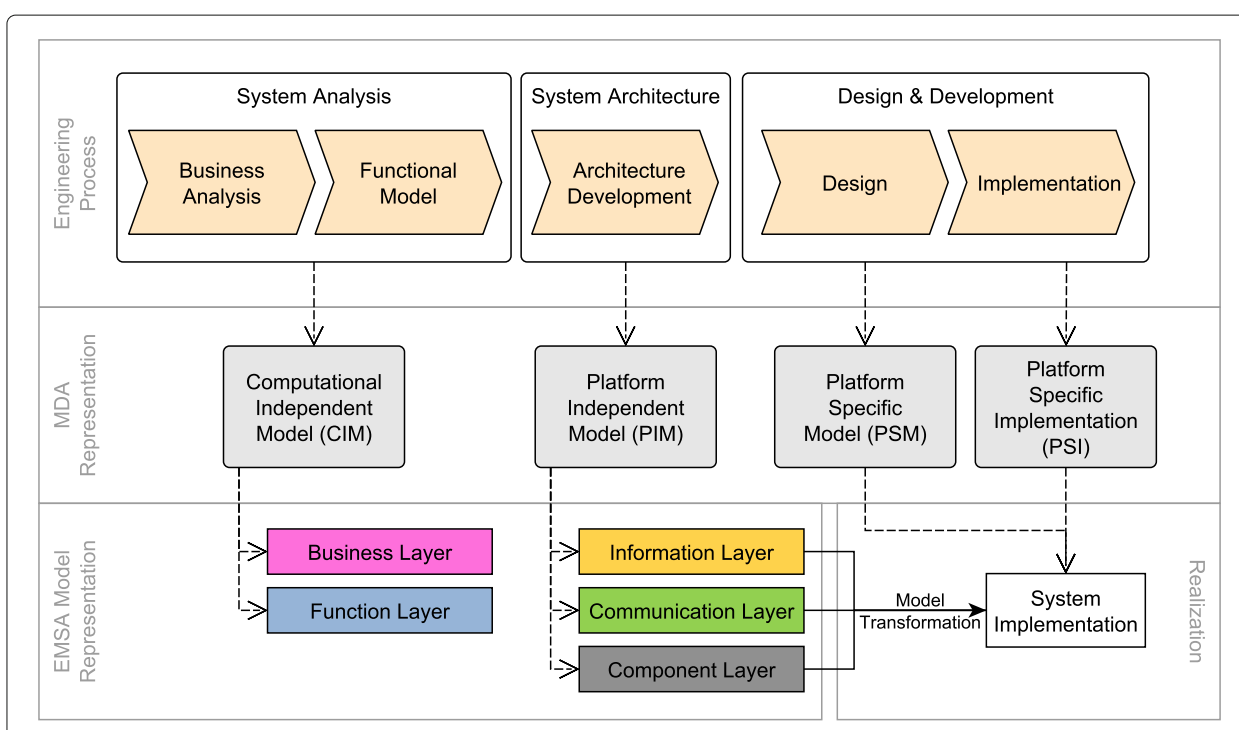

Fig. 9 MDA engineering process mapped to EMSA Model, based on Dänekas et al. (2014); Uslar et al. (2019)

\section{Demonstration of the EMSA in the ELECTRIFIC case study}

The EMSA Model, presented in this paper, has been applied within the context of the EU Horizon2020 research project ELECTRIFIC. The main objective of the project is to seamlessly integrate EVs into the daily life by taking into account the competing goals of different stakeholders (Kirpes et al. 2017; Eider et al. 2017). Among the considered stakeholders are EV user, DSO, EV fleet operator and CS operator. To this end, the main objective of the (1) DSO is to increase the intake of renewable energy as well as ensure certain quality of the provided power, (2) EV fleet operator is to manage its fleet of EVs in terms of optimal charging with respect to costs, percentage of renewable energy, gridand battery-friendliness (e.g to preserve the health of the battery), (3) CS operator is to increase its net profit and (4) EV user is to execute his/her trip plan in the most optimal and efficient manner.

Within the ELECTRIFIC project, in general, we adhere to the granularity and meta model as proposed by Neureiter (2013) for the engineering process in SGAM. This leads to a level of abstraction for ELECTRIFIC as shown in Fig. 10. The following concepts were used:

- Business layer: Business Actors, (Business) Goals, Business Cases

- Function layer: High Level Use Cases (UCs), Primary UCs (=Functions), Function Interrelation

- Information layer: Information Objects, Information Flow

- Communication layer: Communication Protocols and Technologies

- Component layer: Systems, Devices

\section{System modelling on interoperability layers}

A pivotal use case for the ELECTRIFIC system, is the ability to generate and execute a travel plan for the EV user based on his goals. All High Level UCs defined in ELECTRIFIC are the result of iterative discussions and workshops where system engineers and domain experts from different stakeholders have been involved. 


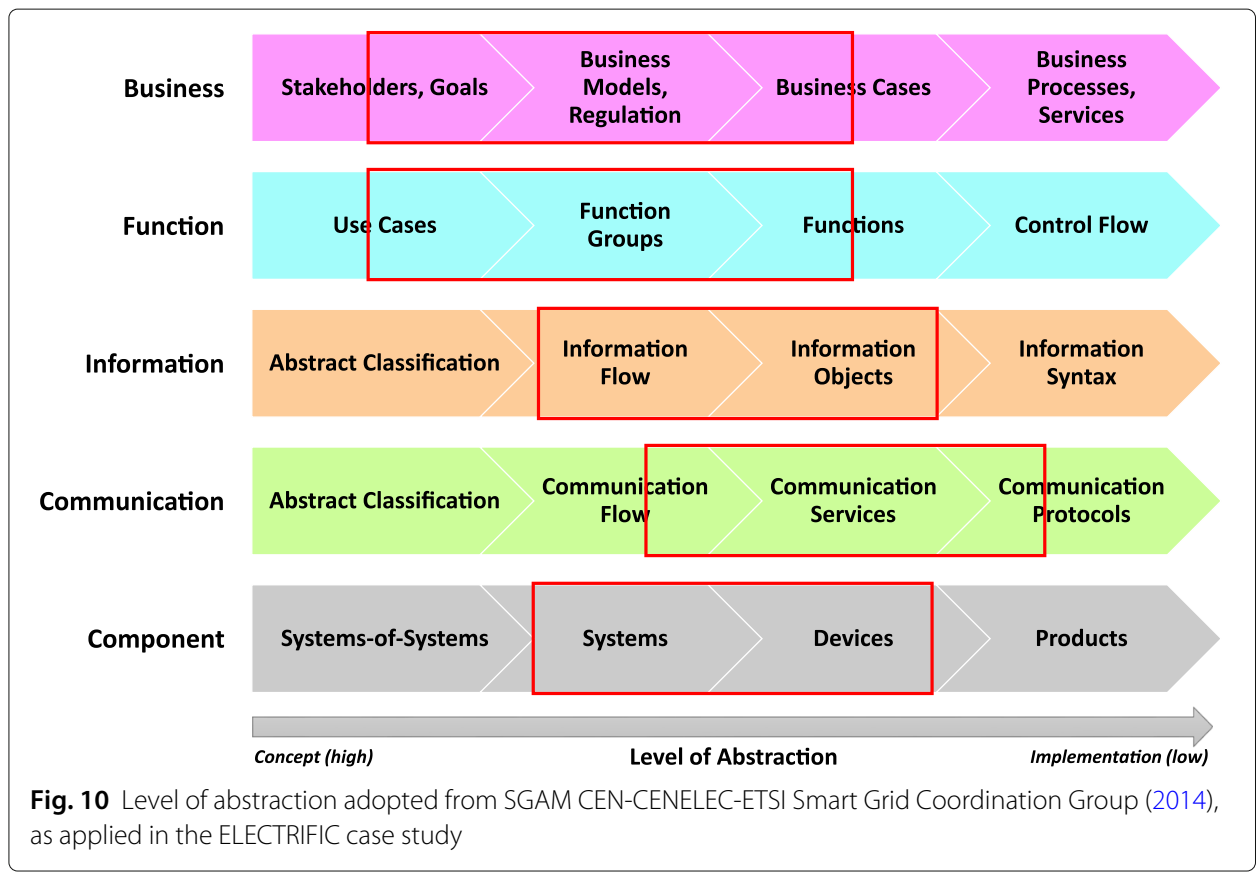

Figure 11 gives a generic overview about this High Level UC on the business layer by illustrating the main business actor, the EV user, its two major goals (improve e-mobility experience and reduce costs from e-mobility), as well as three relevant business cases: reduce range anxiety, improve EV usage behavior, and reduce EV charging costs. This High Level UC is focused only on private EV usage, not for commercial users. Due to space considerations and to keep the use case as simple as possible, we don't consider those other actors and their use cases in this paper.

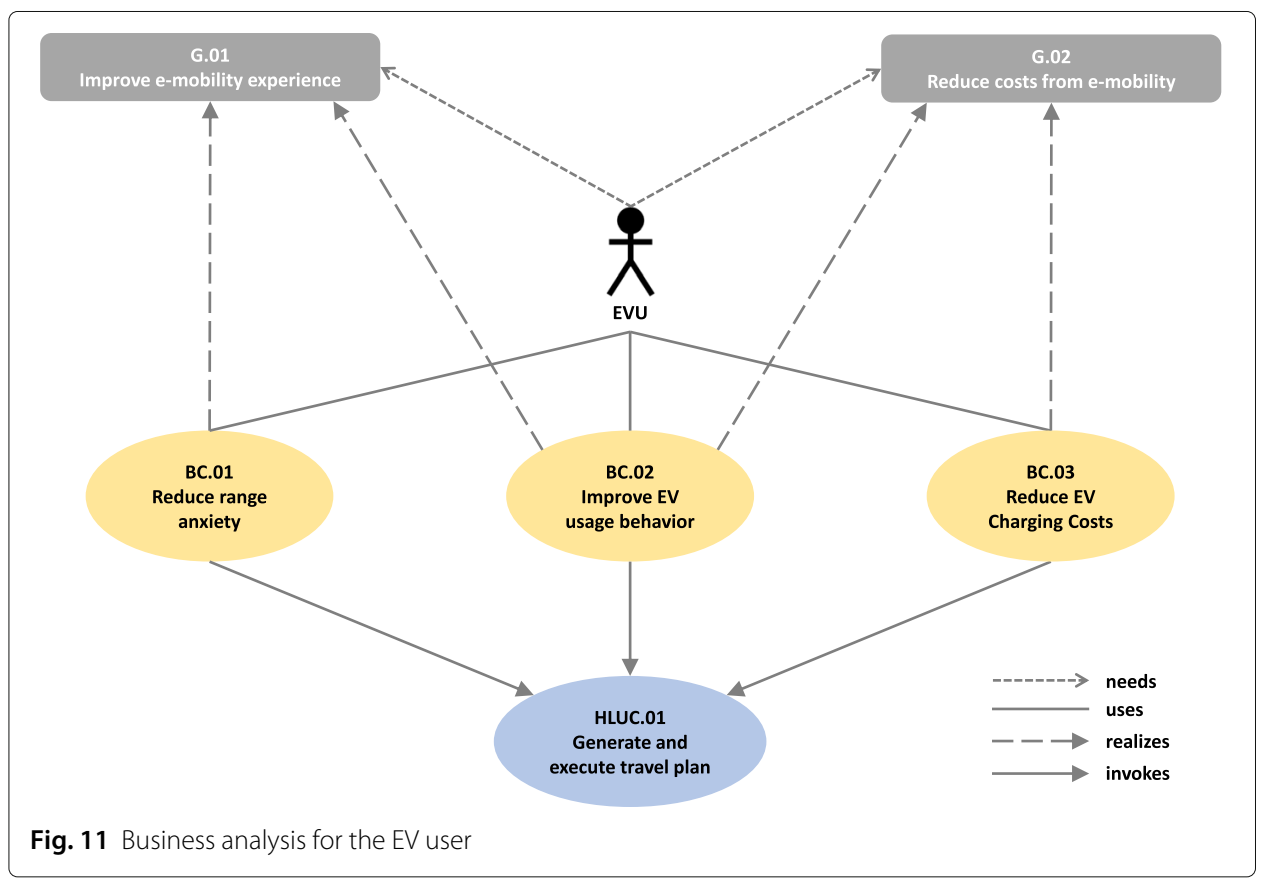


In addition to the business perspective, the corresponding High Level UC can be modelled and visualized with its functional architecture. It gets further decomposed into following three Primary UCs (also called functions) on the function layer (see Fig. 12): (1) retrieval of EV user travel preferences and planning inputs, (2) travel plan creation and optimization and (3) travel plan execution. The realization of the three Primary UCs necessitates functional interrelation and the interaction of the following components on the function layer: ELECTRIFIC App (App), Route-planning service, Charging Station Operator system (CS operator system), Energy-information service, and Battery-health service. To this end, the EV user enters his/her travel preferences into the App. The latter retrieves further travel planning inputs from Route-planning service, CS operator system, Energy-information service and Battery-health service (Primary UC: retrieval of EV user travel preferences and planning inputs). Based on those inputs, the App creates the corresponding travel plans (Primary UC: travel plan creation and optimization). The EV user then reviews the proposed travel plans and can either (1) select a plan for execution, or (2) adjust the input and re-plan another trip or (3) stop interaction with the App. Upon the selection of a travel plan by the EV user, the App performs different tasks (e.g. showing of CS on the map with percentage of renewable energy and navigation) to ensure the execution of the selected plan, and sends back an acknowledgment to the EV user (Primary UC: travel plan execution).

Figure 12 shows an aggregated representation of all EMSA layers (except business layer): the relevant components (component layer), exchanged information (information layer) as well as the communication protocols (communication layer) for this system.

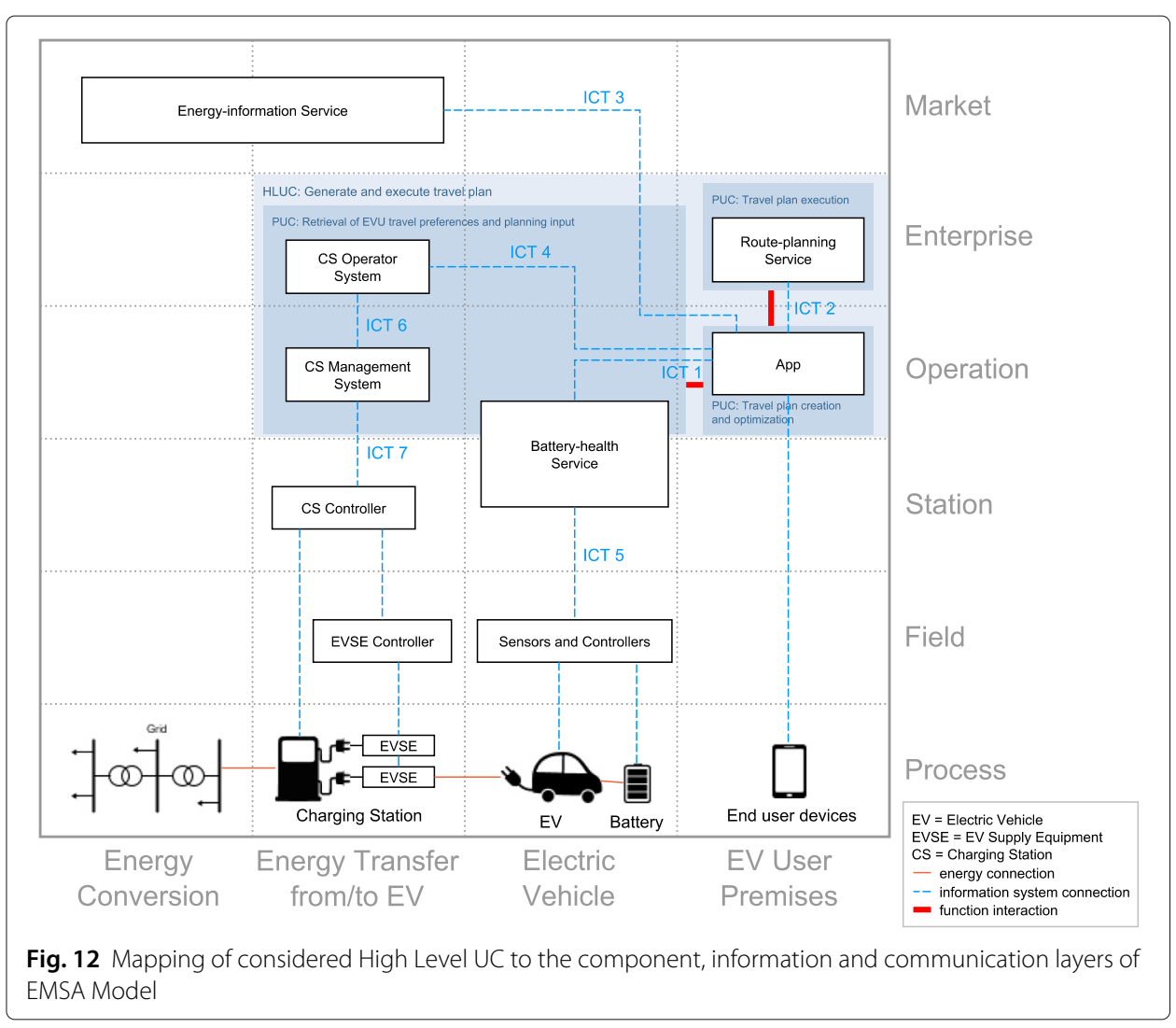


Further details on the information connection (dashed line, colored in blue and labeled with $I C T$ ) are provided in Table 2.

\section{Analysis of data models and protocols}

The first exchange of information (ICT 1) takes place between the App and Battery-health service for providing the EV user with a list of battery health recommendations before the start of any new trip (Table 2). To achieve this, the App sends data about the last trip's driving, parking and charging information to the Battery-health service. The data models for both, request and response between those two components, are proprietary

Table 2 Overview of the exchanged information between different components, data models and communication protocols for the high-level UC

\begin{tabular}{|c|c|c|c|}
\hline $\mathrm{ID}$ & Information Object & Data Model & Protocols \\
\hline \multirow[t]{2}{*}{$\overline{I C T} 1$} & $\begin{array}{l}\text { From App to Battery-health (Request) } \\
\text { Last trip's data for } \\
\text { - Parking: SoC \& ambient temp } \\
\text { - Driving: SoC, discharge rate \& ambient temp } \\
\text { - Charging: SoC, charging rate \& ambient temp }\end{array}$ & Proprietary & HTTP over SSL \\
\hline & $\begin{array}{l}\text { From Battery-health to App (Response) } \\
\text { List of recommendations } \\
\text { - Textual description } \\
\text { - Rationale } \\
\text { - Weighing factor for priorities }\end{array}$ & & $\mathrm{TCP} / \mathrm{IP}$ \\
\hline \multirow[t]{2}{*}{$\mathrm{ICT} 2$} & $\begin{array}{l}\text { From App to Route-planning (Request) } \\
\text { - GPS coordinates of the source and destination }\end{array}$ & Decimal Degrees & HTTP over SSL \\
\hline & $\begin{array}{l}\text { From Route-planning to App (Response) } \\
\text { - List of GPS coordinates needed for navigation } \\
\text { - Traffic Information }\end{array}$ & Proprietary & $\mathrm{TCP} / \mathrm{IP}$ \\
\hline \multirow[t]{2}{*}{ ICT 3} & $\begin{array}{l}\text { From App to Energy-information (Request) } \\
\text { - Country code } \\
\text { - Start and end times }\end{array}$ & ISO 3166-1 ISO 8601 & HTTP over SSL \\
\hline & $\begin{array}{l}\text { From Energy-information to App (Response) } \\
\text { - Percent of renewables } \\
\text { - Energy market price }\end{array}$ & Proprietary & $\mathrm{TCP} / \mathrm{IP}$ \\
\hline \multirow[t]{2}{*}{ ICT 4} & $\begin{array}{l}\text { From App to CS operator system (Request) } \\
\text { - GPS coordinates of a middle point on the trip } \\
\text { - Radius value for Charging Stations filtering }\end{array}$ & Proprietary & HTTP over SSL \\
\hline & $\begin{array}{l}\text { From CS operator system to App (Response) } \\
\text { List of Charging Stations } \\
\text { - GPS coordinates } \\
\text { - Charging Station ID }\end{array}$ & & $\mathrm{TCP} / \mathrm{IP}$ \\
\hline \multirow[t]{2}{*}{ ICT 5} & $\begin{array}{l}\text { From Battery-health to Sensors (Request) } \\
\text { - Vehicle Identification Number }\end{array}$ & ISO 3779 ISO 4030 & HTTP over SSL \\
\hline & $\begin{array}{l}\text { From Sensors to Battery-health (Response) } \\
\text { - State of Charge } \\
\text { - Remaining range }\end{array}$ & Proprietary & $\mathrm{TCP} / \mathrm{IP}$ \\
\hline \multirow[t]{2}{*}{ ICT 6} & $\begin{array}{l}\text { From CS operator sys. to CS manag. sys. (Request) } \\
\text { - Charging Station ID }\end{array}$ & ISO 9834-8 & HTTP over SSL \\
\hline & $\begin{array}{l}\text { From CS manag. sys. to CS operator sys. (Response) } \\
\text { List of static information } \\
\text { - Connector ID, type and standard } \\
\text { - Maximum capacity }\end{array}$ & Proprietary & $\mathrm{TCP} / \mathrm{IP}$ \\
\hline \multirow[t]{2}{*}{ ICT 7} & $\begin{array}{l}\text { From CS manag. sys. to CS Controller (Request) } \\
\text { - Charging Station ID } \\
\text { - Connector ID }\end{array}$ & ISO 9834-8 & HTTP over SSL \\
\hline & $\begin{array}{l}\text { From CS Controller to CS manag. sys. (Response) } \\
\text { - Status information (available) }\end{array}$ & OCPP & $\mathrm{TCP} / \mathrm{IP}$ \\
\hline
\end{tabular}


and data exchange happens using HTTP over SSL communication protocol. To extract the last trip's information which is relevant to driving, parking and charging, information exchange (ICT 5) happens between the Battery-health service and Sensors and Controllers. The former sends the Vehicle Identification Number (which is based on ISO 3779 and ISO 4030 standards) to the latter and receives as a response the (Battery) State of Charge (SoC) and remaining range information directly fetched from the EV. The data model of the response is proprietary and two-way communication happens using HTTP over SSL protocol. Here, the Renault Z.E. Services API has been used, which is modelled and utilized as a Sensors and Controllers component.

The second exchange of information (ICT 2) happens between App and Route-planning service component, where the EV user specifies his/her travel plan by indicating the source and destination of the planned trip. For this purpose, the App sends the GPS coordinates (in the form of Decimal Degrees) to the Route-planning service. It then receives a list of GPS coordinates necessary for the navigation of the considered trip, as well as traffic related information. The data model of the response is proprietary (based on Mapbox) and two-way communication happens using HTTP over SSL protocol.

After receiving the complete list of GPS coordinates from Route-planning service, the App requires to interact with other components. This is necessary for providing the EV user with additional information relevant to the planned trip. To this end, the App sends the GPS coordinates of the location in the middle of the trip as well as a radius value to the CS operator system (ICT 4). The CS operator system identifies all relevant CSs within the range specified. The data models for both, request and response between those two components, are proprietary and exchange happens using HTTP over SSL communication protocol. In order to show the greenness (renewable share) of CSs along the planned trip, the App sends a request to the Energy-information service (ICT 3) by specifying the geographical region (in terms of country code) and the required time period. The data models are based on ISO 3166-1 and ISO 8601 respectively. As a response, the App receives the percentage of the renewable energy (currently on country level) as well as the market energy price. The data model of the response is proprietary and two-way communication happens using HTTP over SSL protocol.

The EV user can select a CS (e.g. based on cheapest or greenness factors) on the planned route. Consequently, the App provides various information (e.g. number of connectors, type of the connectors, status of a connector, etc.) about that specific CS. To achieve this, information need to be exchanged between CS operator system and CS management system (ICT 6) and CS management system and CS controller (ICT 7). Static information of a specific CS can be fetch from CS management system once the CS operator system sends a unique ID (specified by ISO 9834-8 standard) to the latter. The data model of the response is proprietary and two-way communication happens using HTTP over SSL protocol. Dynamic information (e.g. status information) for a specific connector of a CS can be fetch from the CS Controller once the CS management system sends a unique ID (specified by ISO 9834-8 standard) of the CS and the corresponding connector. The data model of the response is based on OCPP using TCP/IP.

\section{Summary of the interoperability analysis}

From Table 2, on the one hand it can be noticed that for this system most of the information objects sent as a request are based on standard data models. The only information 
object, which does not follow any standard data model, is ICT 1 (request). Hence, we assess that there is a need for a standardized data model regarding battery health recommendations. On the other hand, except for ICT 7 (e.g. OCPP), all responses are proprietary, although certain parts of the information objects follow standardized data models like unique identifiers. Despite the fact that a de-facto standard for the exchange of GPS coordinates (e.g. GPX) exists, it is not yet widely adopted. Instead, proprietary data models are preferred. This is the case for the route-planning service (Mapbox) of this system. Consequently, this leads to the conclusion that there is indeed (1) a lack of adoption for standardized data models (e.g. GPX) in the system and (2) a need for data model standards, which hampers the provision of an interoperable system.

\section{Evaluation of the EMSA model}

The evaluation of the final EMSA Model, regarding the identified requirements, is mainly performed qualitatively, realized with an observational case study approach. To this end, we first compare our model with SGAM and then evaluate the fulfillment of the requirements for our framework. We only include the final evaluation, earlier validation on former iterations and feedback cycles with external experts are not considered for this paper.

\section{Evaluative comparison with SGAM}

For a first evaluation of our EMSA Model, we compare it with SGAM by placing all important components of e-mobility into both models' dimensions (Fig. 6 for EMSA Model and Fig. 13 for SGAM): domains and zones. The allocation of the systems in SGAM is aligned with the technical report of the E-Mobility Coordination Group (M/468) and the CEN-CENELEC-ETSI Smart Grid Coordination Group (M/490) (CEN-CENELEC 2015).

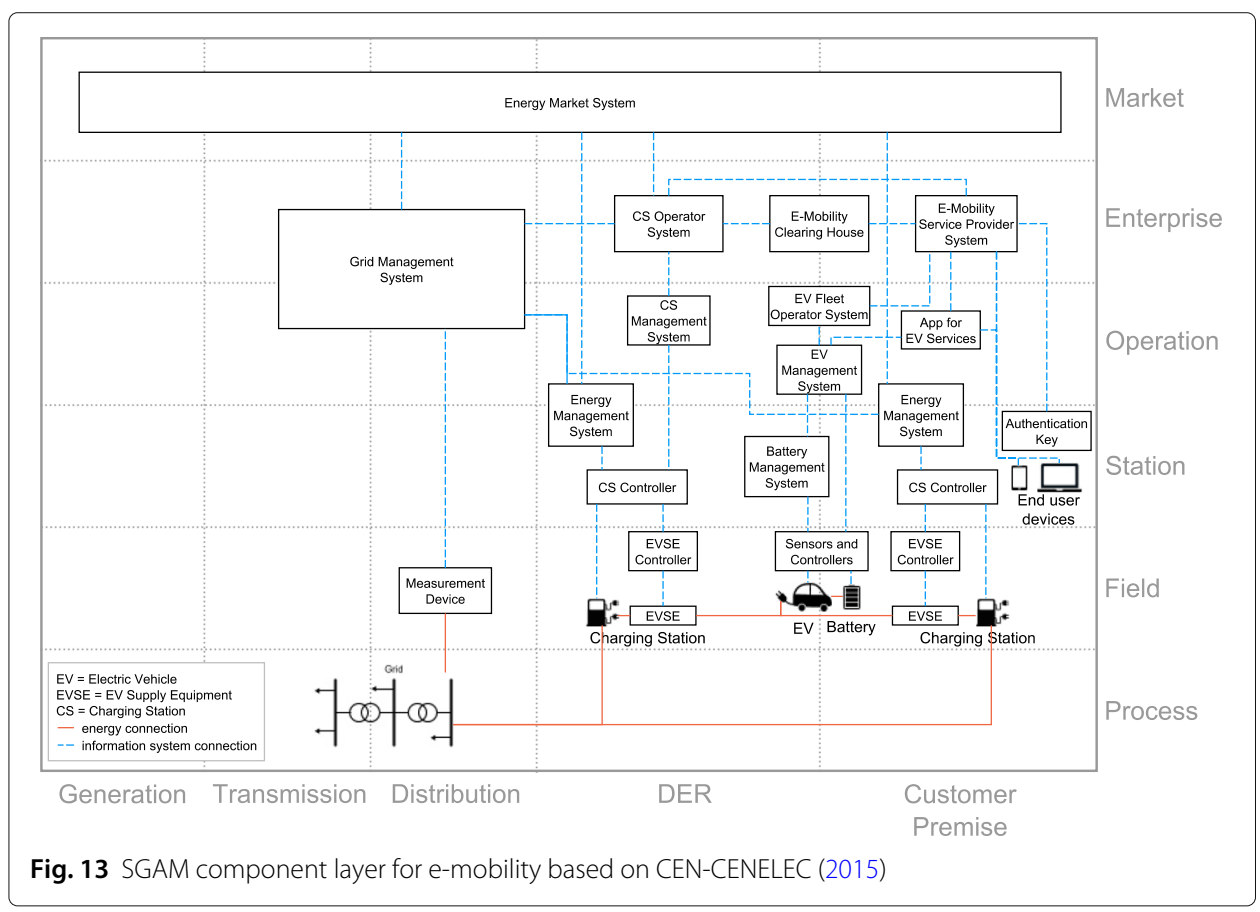


In SGAM, mainly the three domains of Distribution, DER and Customer Premises are utilized. As the focus in SGAM is on the electricity process chain, EVs and CSs are placed in the field zone instead of the process zone. In addition, the EVs and CSs are placed in both domains of DER and Customer Premises, to differentiate between varying grid connection points. In the case of our EMSA Model, both EVs and CSs are handled as separate domains which gives the advantage of allocating components to domains in a clearer fashion, thus giving the possibility to identify gaps even more adequately.

The Grid Management System combines grid balancing and congestion management services. In SGAM the focus is on the grid's point-of-view, whereas in the EMSA Model, the focus is on e-mobility. Here again, the e-mobility process chain (domain division) is better represented in EMSA and the allocation of components to the domains is more clear from e-mobility point-of-view.

In order to quantitatively evaluate the domain separation of our EMSA Model, we placed all ten High Level UCs of the ELECTRIFIC case study into the SGAM as well as into our EMSA Model (Fig. 14). Domain experts of e-mobility and smart grids performed an allocation of the UCs to the domains and zones. As result, a heat map of fields usage frequency of the dimensions has been created.

The focus of the ELECTRIFIC case study is quite grid-related (e.g. smart charging on distribution grid with power quality and grid congestion constraints (Alyousef et al. 2018), dynamic prices for grid-friendly charging). But it also includes battery health recommendations, EV fleet usage and advanced charging services like reservation, dynamic prices and green charging (e.g. renewable share of the energy mix). Concerning the domains in SGAM, all these use cases can therefore be found in the Distribution and DER domain. Some use cases also include the Customer Premises. In the EMSA Model, the use cases are more evenly spread along the available domains, although, there is a tendency to Energy Conversion, Energy Transfer from/to EV and EV User Premises domains.

Concerning the distribution of the High Level UCs over the zones, the difference is quite small. This is due to the fact that the EMSA Model reuses the same six zones from SGAM, which have been adapted to fit the e-mobility point of view. The average usage of

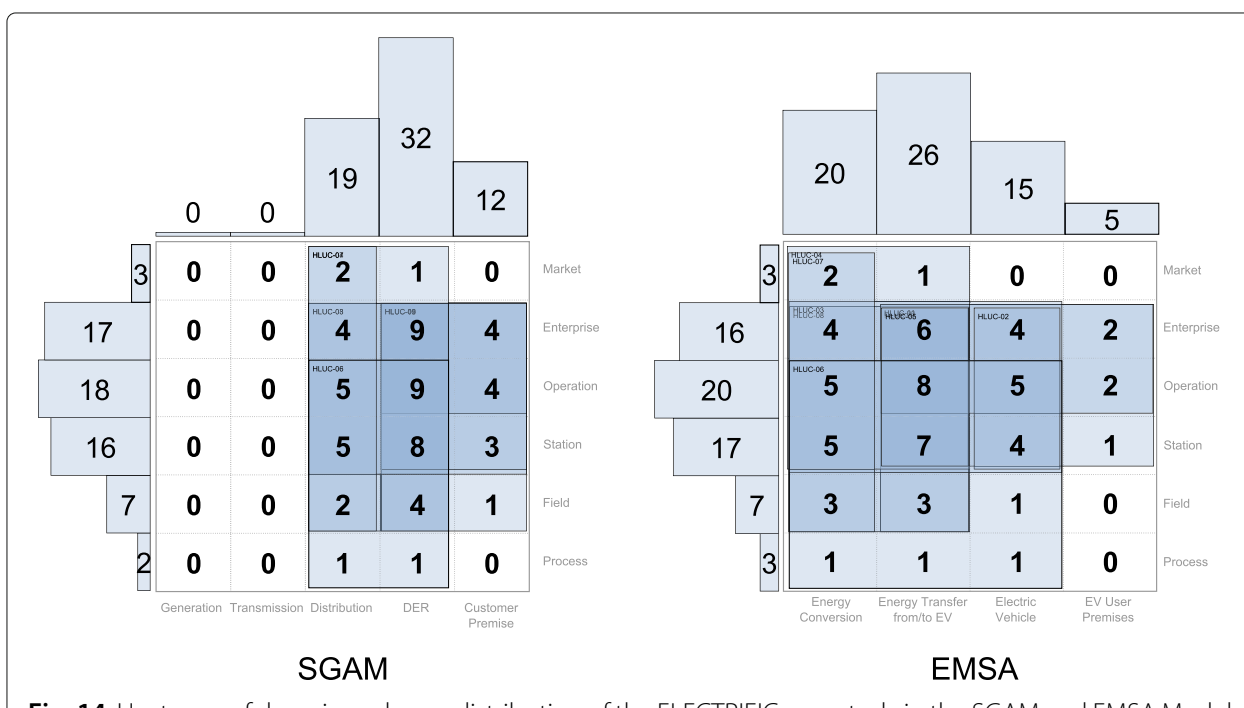

Fig. 14 Heat map of domain and zone distribution of the ELECTRIFIC case study in the SGAM and EMSA Model 
a certain domain-zone field in SGAM is 2.1 and in the EMSA Model 2.75, which indicates a better fitting domain separation of the EMSA Model for the use cases in the case study of ELECTRIFIC. In order to generalize this statement, additional use cases (e.g. public charging stations, wallbox and home charging) would need to be allocated and compared in both models in the future.

\section{Validation of the EMSA regarding complexity and interoperability requirements}

In this section, the fulfillment of Requirement 1 (Provide means to manage and reduce e-mobility systems complexity) and Requirement 2 (Provide means to analyze and ensure e-mobility systems interoperability) regarding their sub-requirements (=objectives) is discussed and evaluated. We provide reference to the relevant design principles and evaluate (1) the implementation of each objective in the EMSA and (2) the demonstration within the case study.

Req1.1: Abstraction and conceptualization The relevant design principles that contribute to the fulfillment of this requirement by the EMSA are Principle 2 (MultiDimensional Structure) and Principle 3 (Allocation, Localization and Consistency). Abstraction and conceptualization are enabled and implemented in the EMSA by utilization of a model-based approach over multiple layers. Further, the level of abstraction can be varied and hierarchical systems with different granularity of sub-systems are considered. The consistent allocation of entities in the EMSA additionally supports these concepts as shown in the comparison with SGAM. In the case study, it has been demonstrated that the means for modelling the systems are sufficient to reduce the complexity and successfully engineer the desired system. The proposed MDA approach may further enhance the abstraction and conceptualization on different layers. This needs to be evaluated in future work.

Req1.2: Separation of concerns The relevant design principle that contributes to the fulfillment of this requirement by the EMSA is Principle 2 (Multi-Dimensional Structure). Separation of concerns is implemented and enacted by the EMSA through integration of different architecture viewpoints. This is mainly achieved with the five interoperability layers, which allow for different perspectives on the architecture of an e-mobility system. In the case study, it has been demonstrated that the separation of concerns in the EMSA is appropriate. When working with the various domain experts from different business units, reduction of the complexity by only considering one or two layers of the system proved to be very useful. The system was successfully engineered, appropriately reflecting the needs of all stakeholders.

Req1.3: Re-usability of components The relevant design principles that contribute to the fulfillment of this requirement by the EMSA are Principle 1 (Scope and Applicability), Principle 3 (Allocation, Localization and Consistency) and Principle 4 (Universality and Flexibility). Re-usability of components is implemented and ensured by applying standardization and harmonization means on all layers. Using standards such as ISO 15118 or IEC 61851 and mapping them to the appropriate location in the EMSA, facilitates allocation and subsequently supports the re-usability of components and systems. In the case study, it has been demonstrated that re-usability of services and sub-systems 
is an important feature to reduce complexity of a system. Many of the ELECTRIFIC services and components are used by multiple systems, their alignment is ensured by the EMSA Model.

Req1.4: Complexity reduction of the engineering process The relevant design principles that contribute to the partial fulfillment of this requirement by the EMSA are Principle 2 (Multi-Dimensional Structure) and Principle 3 (Allocation, Localization and Consistency). Complexity reduction of the engineering process is implemented by the EMSA framework with the inclusion of a very brief guidance and documentation. In future work, this needs to be further enhanced by providing a sophisticated and comprehensive engineering process with guidance. In the case study, it has been demonstrated that the EMSA methodology indeed reduces complexity of the engineering process, especially for the domain experts and the system engineers. This could probably be further improved by applying the suggested MDA approach, which works well with the SGAM.

Req2.1: Analysis and assessment of interoperability The relevant design principles that contribute to the fulfillment of this requirement by the EMSA are Principle 2 (MultiDimensional Structure) and Principle 3 (Allocation, Localization and Consistency). The interoperability assessment of systems is implemented by providing an allocation of communication and information standards on the respective layers. In the case study, it has been demonstrated that the EMSA has successfully been used to analyze the interoperability of the ELECTRIFIC system considered for that respective use case. By conducting multiple iterations of the assessment, potential interoperability issues of this system have been eliminated during the project.

Req2.2: Identification of standardization gaps The relevant design principles that contribute to the fulfillment of this requirement by the EMSA are Principle 2 (MultiDimensional Structure) and Principle 3 (Allocation, Localization and Consistency). Similar to $\operatorname{Req}_{2.1}$, the identification of gaps in standardization is implemented by allocating all existing relevant standards to the respective layers (information and communication). In the case study, it has been demonstrated that the EMSA Model can be utilized to identify such gaps on different layers. If for an exchanged information object between two components, a proprietary data model is used this is either 1) an interoperability issue within the system or 2) a gap in standardization. This can be analyzed with the EMSA. Future created standards need to be mapped to the respective domain/zone on the appropriate layer.

With the demonstration in the ELECTRIFIC case study and its evaluation, we show that most of the requirements are completely fulfilled. Due to the limitations of a journal paper, Req1.4 (Complexity reduction of the engineering process) is only partially fulfilled. Provision of further guidance on the engineering process and additional documentation is out of scope for this research work, but will be considered in future work.

\section{Conclusion and outlook}

A suitable design and engineering process for ISs is required to align use cases and business objectives with the functional and technological requirements of a system. This is especially important for sector-coupled applications like e-mobility, which combines 
aspects from the energy and the mobility sector. Typically, the complexity of system architectures is reduced through abstraction, conceptualization and other means. For the energy sector, SGAM has been proposed as a systems architecture model to facilitate the design and development of ISs in the context of power systems. Inspired by SGAM, several independent approaches with a similar purpose in the context of smart cities or e-mobility have been proposed. We showed, that none of the existing approaches satisfies all of the requirements adequately. Subsequently, we designed and developed the E-Mobility Systems Architecture (EMSA) model with multiple iterations and in this paper, introduced the final model which is based on the extracted design principles. The EMSA is suitable for application with systems of the whole e-mobility sector. A case study is introduced, which has been conducted during the ELECTRIFIC project to demonstrate utility of the EMSA Model and in order to assess and validate it against the above-mentioned requirements. We find, that our systems architecture model fulfills all requirements sufficiently. Further, we provide some guidance on the usage of the EMSA Model for research-oriented as well as for practical applications. It supports the users of the model, namely domain and system engineering experts, typically from industry in their complex tasks of analyzing existing and building new interoperable systems for e-mobility. However, the description and validation of our model is limited to battery electric vehicles.

The evaluation of the domains and zones definitions compared to SGAM serves as an outlook for future research. Further research activities could be conducted, e.g. an enhanced quantitative comparison of the related approaches with the EMSA Model, by considering a larger and harmonized set of e-mobility related use cases. Due to the dynamically evolving e-mobility ecosystem, standardization activities are still ongoing. Future released standards, such as IEC 61980, IEC 63110 or IEC 63119 need to be mapped to the respective location. In future work, the EMSA Model might be extended to a complete framework including an engineering process, more guidance and documentation and a reference architecture for e-mobility information systems.

\begin{abstract}
Abbreviations
BMS: Battery Management System; CIM: Computational Independent Model; CS: Charging Station; DER: Distributed Electrical Resource; DSL: Domain-Specific Language; DSO: Distribution System Operator; DSR: Design Science Research; EM-ISA: E-Mobility Information System Architecture; EMAM: E-Mobility Architecture Model; EMS: Energy Management System; EMSA: E-Mobility Systems Architecture; EV: Electric Vehicle; GSCAM: Generic Smart City Architecture Model; GWAC: GridWise Architecture Council; ICT: Information and Communications Technology; IS: Information System; MDA: Model-Driven Architecture; PIM: Platform Independent Model; PSI: Platform Specific Implementation; PSM: Platform Specific Model; PWM: Pulse Width Modulation; SCIAM: Smart City Infrastructure Architecture Model; SGAM: Smart Grid Architecture Model; SoC: (Battery) State of Charge; SysML: Systems Modeling Language; TSO: Transmission System Operator; UC: Use Case; UML: Unified Modeling Language
\end{abstract}

Acknowledgements

We would like to thank all ELECTRIFIC project partners for the fruitful discussions and their valuable feedback during design, development and evaluation of the EMSA model.

\title{
Authors' contributions
}

BK did main research on related work and wrote the corresponding sections. PD and BK were mainly involved in creating the EMSA Model, especially the layers, zones and domains definition. RB detailed the High Level UC of the case study done within the ELECTRIFIC project. The comparison of SGAM and EMSA qualitatively on component and quantitatively on function layer was mainly performed by PD with the help of BK. The validation section was done by BK. HdM and CB contributed in conception and revision of the EMSA Model and the whole paper. All authors have read and approved the final manuscript.

Funding

This project has received funding from the European Union's Horizon 2020 research and innovation programme under grant agreement No. 713864 (ELECTRIFIC). 


\section{Availability of data and materials}

The detailed description of the use cases which have been used and/or analyzed within the case study are available from the corresponding author on reasonable request and will also be published on the project's website (https://electrific.eu/).

\section{Competing interests}

The authors declare that they have no competing interests.

\section{Author details}

${ }^{1}$ University of Mannheim, Schloss, 68131 Mannheim, Germany. ${ }^{2}$ Bayernwerk AG, Lilienthalstraße 7, 93049 Regensburg, Germany. ${ }^{3}$ University of Passau, Innstraße 41, 94032 Passau, Germany.

Received: 22 March 2019 Accepted: 18 July 2019

Published online: 28 August 2019

\section{References}

Alyousef A, Danner D, Kupzog F, Meer HD (2018) Enhancing power quality in electrical distribution systems using a smart charging architecture. Energy Inform 1(1):28

Benbya H, Mckelvey B (2006) Toward a complexity theory of information systems development. Inf Technol People 19(1):12-34

Brand A, lacob M-E, van Sinderen MJ (2015) Interoperability Architecture for Electric Mobility. In: van Sinderen M, Chapurlat V (eds). Enterprise Interoperability. Springer, Berlin, Heidelberg. pp 126-140

Brown S, Pyke D, Steenhof P (2010) Electric vehicles: The role and importance of standards in an emerging market. Energy Policy 38(7):3797-3806

CEN-CENELEC (2015) Report About Smart Charging of Electric Vehicles in Relation to Smart Grid E-Mobility Smart Charging. ftp://ftp.cen.eu/EN/EuropeanStandardization/HotTopics/ElectricVehicles/SmartChargingReport.pdf. Accessed 31 July 2019

CEN-CENELEC-ETSI Smart Grid Coordination Group (2012) Smart Grid Reference Architecture. https://ec.europa.eu/ energy/sites/ener/files/documents/xpert_group1_reference_architecture.pdf. Accessed 31 July 2019

CEN-CENELEC-ETSI Smart Grid Coordination Group (2014) SG-CG/M490/K_SGAM Usage and Examples. ftp://ftp.cencenelec. eu/EN/EuropeanStandardization/HotTopics/SmartGrids/SGCG_Methodology_SGAMUserManual.pdf. Accessed 31 July 2019

Courtney J, Merali Y, Paradice D, Wynn E (2008) On the Study of Complexity in Information Systems. Int J Inform Technol Syst Approach 1(1):37-48

Dänekas C., Neureiter C, Rohjans S, Uslar M, Engel D (2014) Towards a Model-Driven-Architecture Process for Smart Grid Projects. In: Benghozi P, Krob D, Lonjon A, Panetto H (eds). Digital Enterprise Design \& Management. Springer, Cham. pp 47-58

Danner P, Duschl W, Danner D, Alyousef A, de Meer H (2018) Flexibility Reward Scheme for Grid-Friendly Electric Vehicle Charging in the Distribution Power Grid. In: Proceedings of the 3th Workshop on Electric Vehicle Systems, Data, and Applications of EV-Sys '18. ACM, New York. pp 1-6

DKE/DIN/NDE (2014) The German Standardization Roadmap Smart City. Technical report, DIN e.V. and DKE Deutsche Kommission Elektrotechnik Elektronik Informationstechnik im DIN und VDE

Eider M, Stolba M, Sellner D, Berl A, Basmadjian R, de Meer H, Klingert S, Schulze T, Kutzner F, Kacperski C (2017) Seamless Electromobility. In: Proceedings of the Eighth International Conference on Future Energy Systems - e-Energy '17. pp 316-321. http://dl.acm.org/citation.cfm?doid=3077839.3078461. Accessed 31 July 2019

ElaadNL (2017) EV Related Protocol Study v1.1. Technical report, ElaadNL

Electrific Consortium (2019) Electrific Project. https://electrific.eu/. Accessed 31 July 2019

European Commission (2019) Database of European projects. https://cordis.europa.eu/projects/en. Accessed 31 July 2019

Geraci A, Katki F, McMonegal L, Meyer B, Lane J, Wilson P, Radatz J, Yee M, Porteous H, Springsteel F (1991) IEEE standard computer dictionary: Compilation of IEEE standard computer glossaries. IEEE Press, Piscataway

GWAC (2008) Gridwise Interoperability Context-Setting Framework. https://www.gridwiseac.org/pdfs/ interopframework_v1_1.pdf

Hevner AR, March ST, Park J, Ram S (2004) Design Science in Information Systems Research. MIS Q 28(1):75-105

Holub I (2016) Methodology for Measuring the Complexity of Enterprise Information Systems. J Syst Integr 7(3):34-53

Honour E (2006) Systems Engineering and Complexity. In: Proceedings of the Conference on Systems Engineering Research. INCOSE, San Diego

ISO Central Secretary (2015) Systems and software engineering - System life cycle processes, ISO/IEC/IEEE 15288:2015, Geneva

ISO Central Secretary (2016) Road vehicles - Vehicle-to-Grid Communication Interface - Part 2: Network and application protocol requirements, ISO/DIS 15118-2, Geneva

ISO, IEC, IEEE (2011) International Standard ISO/IEC/IEEE 42010:2011 Systems and software engineering - Architecture Description. http://ieeexplore.ieee.org/stamp/stamp.jsp?tp=\&arnumber=6146379\&isnumber=6146378

King JL, Simon CP (2015) Complications with Complexity in Requirements. ACM Trans Manag Inf Syst 5(3):1-12

Kirpes B, Becker C (2018) Processing Electric Vehicle Charging Transactions in a Blockchain-based Information System. In: 24th Americas Conference on Information Systems (AMCIS 2018). Association for Information Systems (AIS), New Orleans. pp 1-5

Kirpes B, Klingert S, Basmadjian R, de Meer H, Eider M, Perez Ortega M (2017) EV Charging Coordination to secure Power Grid Stability. In: 1st E-Mobility Power System Integration Symposium (Berlin). Association for Information Systems, Berlin

Kossahl J, Busse S, Kolbe LM (2012) The Evolvement of Energy Informatics in the Information Systems Community - A literature Analysis and Research Agenda. In: 20th European Conference on Information Systems, ECIS 2012, Barcelona, Spain, June 10-13, 2012. p 172. http://aisel.aisnet.org/ecis2012/172. Accessed 31 July 2019 
Nationaal Kennisplatform Laadinfrastructuur, Robert and Philips, Simon and Zwirello, Chris and Schilling, Simon and Warnier, Olger (2019) OCPI 2.2-RC1. https://github.com/ocpi

Lemberger PP, Morel M (2012) Managing Complexity of Information Systems: The Value of Simplicity. Wiley, Hoboken Lopes AJ, Lezama R, Pineda R (2011) Model Based Systems Engineering for Smart Grids as Systems of Systems. In: Procedia Comput Sci Vol. 6. pp 441-450

Neureiter C (2013) Introduction to the "SGAM Toolbox". Tech. Rep.

Neureiter C, Engel D, Uslar M (2016) Domain Specific and Model Based Systems Engineering in the Smart Grid as Prerequesite for Security by Design. Electronics 5(2):24

Neureiter C, Rohjans S, Engel D, Dänekas C, Uslar M (2014) Addressing the Complexity of Distributed Smart City Systems by Utilization of Model Driven Engineering Concepts. In: VDE-Kongress 2014. VDE, Frankfurt/Main. pp 1-6

Object Management Group (2014) MDA Guide rev. 2.0. Technical Report June, Object Management Group. http://www. omg.org/cgi-bin/doc?ormsc/14-06-01

Open Charge Alliance (2018) OCPP 2.0 Public Draft document. https://www.openchargealliance.org

Peffers K, Tuunanen T, Rothenberger MA, Chatterjee S (2008) A Design Science Research Methodology for Information Systems Research. J Manag Inform Syst 24(3):45-77

Portela CM, Klapwijk P, Verheijen L, de Boer H, Slootweg H, van Eekelen M (2015) OSCP - An open protocol for smart charging of electric vehicles. CIRED

Ranganathan A, Campbell R (2007) What is the Complexity of a Distributed Computing System?. Complexity 12(6):37-45 Rodríguez R, Madina C, Zabala E (2015) EV Integration in Smart Grids Through Interoperability Solutions. EVS28 Int Electric Veh Symp Exhibition:1-12

Schmidt J, Busse S (2013) The Value of IS to Ensure the Security of Energy Supply - The Case of Electric Vehicle Charging In: 19th AMCIS Proceedings. Association for Information Systems, Chicago, Illinois. pp 1-9

Schmutzler J, Andersen CA, Wietfeld C (2013) Evaluation of OCPP and IEC 61850 for smart charging electric vehicles. World Electric Veh J 6(4):863-874

Schuh G, Fluhr J, Birkmeier M, Sund M (2013) Information system architecture for the interaction of electric vehicles with the power grid. In: 2013 10th IEEE International Conference on Networking, Sensing and Control (ICNSC). IEEE, Evry, France. pp 821-825. http://ieeexplore.ieee.org/document/6548844/

Uslar M, Engel D (2015) Towards Generic Domain Reference Designation: How to learn from Smart Grid Interoperability. In: D-A-CH Energieinformatik. KIT

Uslar M, Gottschalk M (2015) Extending the SGAM for Electric Vehicles Vol. 95. pp 219-226

Uslar M, Rohjans S, Neureiter C, Pröstl Andrén F, Velasquez J, Steinbrink C, Efthymiou V, Migliavacca G, Horsmanheimo S, Brunner H, Strasser T (2019) Applying the Smart Grid Architecture Model for Designing and Validating System-of-Systems in the Power and Energy Domain: A European Perspective. Energies 12(2):258

Uslar M, Trefke J (2014) Applying the Smart Grid Architecture Model SGAM to the EV Domain. In: Proceedings of the 28th Envirolnfo 2014 Conference. BIS-Verlag, Oldenburg. pp 821-826

\section{Publisher's Note}

Springer Nature remains neutral with regard to jurisdictional claims in published maps and institutional affiliations.

\section{Submit your manuscript to a SpringerOpen ${ }^{\circ}$ journal and benefit from:}

$\checkmark$ Convenient online submission

- Rigorous peer review

- Immediate publication on acceptance

- Open access: articles freely available online

- High visibility within the field

Retaining the copyright to your article

Submit your next manuscript at $\gg$ springeropen.com 